\title{
Hyperplane arrangements and diagonal harmonics
}

\author{
Drew Armstrong*
}

In 2003, Haglund's bounce statistic gave the first combinatorial interpretation of the $q, t$-Catalan numbers and the Hilbert series of diagonal harmonics. In this paper we propose a new combinatorial interpretation in terms of the affine Weyl group of type $A$. In particular, we define two statistics on affine permutations; one in terms of the Shi hyperplane arrangement, and one in terms of a new arrangement - which we call the Ish arrangement. We prove that our statistics are equivalent to the area' and bounce statistics of Haglund and Loehr. In this setting, we observe that bounce is naturally expressed as a statistic on the root lattice. We extend our statistics in two directions: to "extended" Shi arrangements and to the bounded chambers of these arrangements. This leads to a (conjectural) combinatorial interpretation for all integral powers of the Bergeron-Garsia nabla operator applied to elementary symmetric functions.

AMS 2000 SUBJECT CLASSIFICATIONS: 05E10, 52C35.

KEYWORDS AND PHRASES: Shi arrangement, Ish arrangement, affine permutations, diagonal harmonics, Catalan numbers, nabla operator, parking functions.

\section{Introduction}

First we define the diagonal harmonics - which we will keep in mind throughout - then we discuss hyperplane arrangements - which the paper is really about.

\subsection{Diagonal harmonics}

The symmetric group $\mathfrak{S}(n)$ acts on the polynomial ring $S=\mathbb{Q}\left[x_{1}, \ldots, x_{n}\right]$ by permuting variables. Newton showed that the subring of $\mathfrak{S}(n)$-invariant polynomials is generated by the algebraically independent power sum polynomials: $p_{k}=\sum_{i=1}^{n} x_{i}^{k}$ for $k=1,2, \ldots, n$. It is known that the coinvariant

arXiv: 1005.1949

*Partially supported by NSF award DMS-1001825. 
ring $R=S /\left(p_{1}, \ldots, p_{n}\right)$ is a graded version of the regular representation of $\mathfrak{S}(n)$, with Hilbert series

$$
\sum_{i=0}^{n} \operatorname{dim} R_{i} q^{i}=\prod_{j=1}^{n}\left(1+q+q^{2}+\cdots+q^{j}\right)=[n]_{q} ! .
$$

The dual ring $S^{*}=\mathbb{Q}\left[\partial / \partial x_{1}, \ldots, \partial / \partial x_{n}\right]$ acts on $S$ via the pairing $\left(\partial / \partial x_{i}\right) x_{j}=\delta_{i j}$, hence the coinvariant ring is isomorphic to the quotient $S^{*} /\left(p_{1}^{*}, \ldots, p_{n}^{*}\right)$, where $p_{k}^{*}=\sum_{i=1}^{n}\left(\partial / \partial x_{i}\right)^{k}$ for $k=1, \ldots, n$. On the other hand, this quotient is naturally isomorphic to the submodule $H \subseteq S$ annihilated by the $p_{k}^{*}$ :

$$
H=\left\{f \in S: p_{k}^{*} f=0 \text { for all } k\right\} .
$$

This $H$ is called the ring of harmonic polynomials since, in particular, $p_{2}^{*}$ is the standard Laplacian operator on $S$.

Now consider the ring $D S=\mathbb{Q}\left[x_{1}, \ldots, x_{n}, y_{1}, \ldots, y_{n}\right]$ of polynomials in two sets of commuting variables, together with the diagonal action of $\mathfrak{S}(n)$, which permutes the $x$ variables and the $y$ variables simultaneously. Weyl [31] showed that the $\mathfrak{S}(n)$-invariant subring of $D S$ is generated by the polarized power sums: $p_{k, \ell}=\sum_{i=1}^{n} x_{i}^{k} y_{i}^{\ell}$ for all $k+\ell>0$. Hence the ring of diagonal coinvariants $D R=D S /\left(p_{k, \ell}: k+\ell>0\right)$ is naturally isomorphic to the ring of diagonal harmonic polynomials:

$$
D H=\left\{f \in D S: \sum_{i=1}^{n}\left(\partial / \partial x_{i}\right)^{k}\left(\partial / \partial y_{i}\right)^{\ell} f=0 \text { for all } k+\ell>0\right\} .
$$

The diagonal action preserves the bigrading of $D S$ by $x$-degree and $y$-degree, hence $D H$ is a bigraded $\mathfrak{S}(n)$-module. The bigraded Hilbert series

$$
\mathcal{D H}(n ; q, t):=\sum_{i, j=0}^{n} \operatorname{dim}(D H)_{i, j} q^{i} t^{j}
$$

has beautiful and remarkable properties. The study of $\mathcal{D H}(n ; q, t)$ was initiated by Garsia and Haiman (see [13]) and is today an active area of research.

\subsection{Some arrangements}

Let $\left\{e_{1}, e_{2}, \ldots, e_{n}\right\}$ be the standard basis for $\mathbb{R}^{n}$. Given $v \in \mathbb{R}^{n}$ and $k \in$ $\mathbb{R}$, we will often use the notation " $v=k$ " as shorthand for the set $\{x$ : 


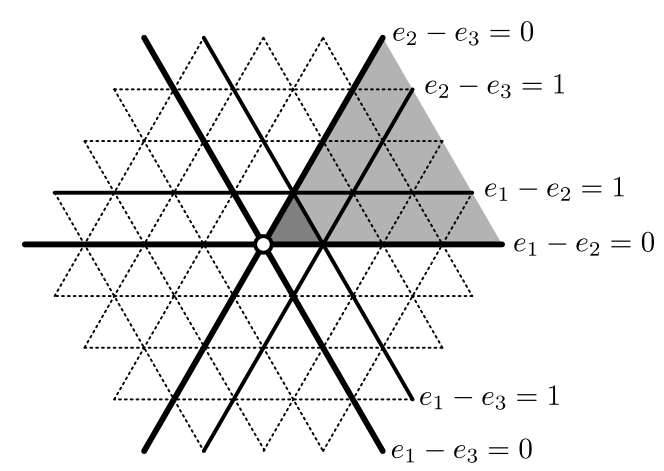

Figure 1.1: Some arrangements in $\mathbb{R}_{0}^{3}$.

$(x, v)=k\} \subseteq \mathbb{R}^{n}$, where $(\cdot, \cdot)$ is the standard inner product. Consider the following three arrangements of hyperplanes, respectively called the Coxeter arrangement, Shi arrangement, and affine arrangement of type $A_{n-1}$ :

$$
\begin{aligned}
\operatorname{Cox}(n) & :=\left\{e_{i}-e_{j}=a: 1 \leq i<j \leq n, a=0\right\}, \\
\operatorname{Shi}(n): & =\left\{e_{i}-e_{j}=a: 1 \leq i<j \leq n, a \in\{0,1\}\right\}, \\
\operatorname{Aff}(n): & =\left\{e_{i}-e_{j}=a: 1 \leq i<j \leq n, a \in \mathbb{Z}\right\} .
\end{aligned}
$$

Since all hyperplanes in this paper contain the line $e_{1}+e_{2}+\cdots+e_{n}$, we will typically think of these arrangements in the $(n-1)$-dimensional space

$$
\mathbb{R}_{0}^{n}:=\left\{e_{1}+e_{2}+\cdots+e_{n}=0\right\} .
$$

If $\mathcal{A}$ is an arrangement in a space $V$ then the connected components of the complement $V-\cup_{H \in \mathcal{A}} H$ are called chambers. We will refer to chambers of the Coxeter arrangement as cones; and refer to affine chambers as alcoves. Let $C_{\circ}$ denote the dominant cone, which satisfies the coordinate inequalities

$$
e_{1}>e_{2}>\cdots>e_{n}
$$

and let $A_{\circ}$ denote the fundamental alcove, satisfying

$$
e_{1}>e_{2}>\cdots>e_{n}>e_{1}-1 .
$$

Figure 1.1 displays the arrangements $\operatorname{Cox}(3)$, Shi(3), and Aff(3) in $\mathbb{R}_{0}^{3}$, with the dominant cone and fundamental alcove shaded. The Shi arrangement was introduced by Jian-Yi Shi (see [23, Chapter 7]) in his description of the Kazhdan-Lusztig cells for certain affine Weyl groups. 


\subsection{Symmetric group}

The symmetric group $\mathfrak{S}(n)$ has a faithful representation as a group of isometries of $\mathbb{R}_{0}^{n}$ generated by the set

$$
S=\left\{s_{1}, s_{2}, \ldots, s_{n-1}\right\},
$$

where $s_{i}$ is the reflection in the hyperplane $e_{i}-e_{i+1}=0$. The reflection $s_{i}$ corresponds in $\mathfrak{S}(n)$ to the transposition of adjacent symbols $(i, i+1)$.

The symmetric group acts simply-transitively on the cones of the Coxeter arrangement $\operatorname{Cox}(n)$. By convention, let the dominant cone $C_{\circ}$ correspond to the identity permutation; then for any permutation $w \in \mathfrak{S}(n)$ the cone $w C_{\circ}$ satisfies

$$
e_{w(1)}>e_{w(2)}>\cdots>e_{w(n)} .
$$

\subsection{Affine symmetric group}

Now let $s_{n}$ denote the reflection in the affine hyperplane $e_{1}-e_{n}=1$. The linear reflections $\left\{s_{1}, s_{2}, \ldots, s_{n-1}\right\}$ together with the affine reflection $s_{n}$ generate the affine Weyl group of type $\tilde{A}_{n}$. This group acts simply-transitively on the set of alcoves, where the fundamental alcove $A_{\circ}$ corresponds to the identity element of the group. Note that $A_{\circ}$ is a (non-regular) simplex in $\mathbb{R}_{0}^{n}$ whose facets are supported by the reflecting hyperplanes of the generators $\left\{s_{1}, s_{2}, \ldots, s_{n}\right\}$.

Lusztig [21] studied an affine version of the symmetric group, whose combinatorial properties were further developed by Björner and Brenti [5]. We define $\tilde{\mathfrak{S}}(n)$ as the group of infinite permutations $\tilde{w}: \mathbb{Z} \rightarrow \mathbb{Z}$ satisfying:

- $\tilde{w}(k+n)=\tilde{w}(k)+n$ for all $k \in \mathbb{Z}$,

- $\tilde{w}(1)+\tilde{w}(2)+\cdots+\tilde{w}(n)=\left(\begin{array}{c}n+1 \\ 2\end{array}\right)$.

The first property says that $\tilde{w}$ is periodic and the second fixes a frame of reference. The elements of $\tilde{\mathfrak{S}}(n)$ are called affine permutations, and $\tilde{\mathfrak{S}}(n)$ is the affine symmetric group. Following Björner and Brenti, we will usually express an affine permutation $\tilde{w} \in \tilde{\mathfrak{S}}(n)$ using the window notation:

$$
" \tilde{w}=[\tilde{w}(1), \tilde{w}(2), \ldots, \tilde{w}(n)] . "
$$

For integers $i<j$ we will write $((i, j)): \mathbb{Z} \rightarrow \mathbb{Z}$ to denote the "affine transposition" that swaps the elements in positions $i+k n$ and $j+k n$ for all $k \in \mathbb{Z}$. We could also write $((i, j))=\prod_{k}(i+k n, j+k n)$. Lusztig proved 
that the correspondence $s_{i} \leftrightarrow((i, i+1))$ defines an isomorphism between the affine symmetric group and the affine Weyl group of type $\tilde{A}_{n}$. Here the affine transposition $((i, j))$ corresponds to the reflection in the affine hyperplane

$$
e_{i^{\prime}}-e_{j^{\prime}}=\left\lceil\frac{i}{n}\right\rceil-\left\lceil\frac{j}{n}\right\rceil,
$$

where $i^{\prime}=i-n(\lceil i / n\rceil-1)$ and $j^{\prime}=j-n(\lceil j / n\rceil-1)$. In particular, note that the generator $s_{i}=((i, i+1))$ corresponds to $e_{i}-e_{i+1}=0$ for $1 \leq i \leq n-1$, and $s_{n}=((n, n+1))$ corresponds to $e_{1}-e_{n}=1$.

\subsection{The Ish arrangement}

Finally, we introduce a new hyperplane arrangement, called the Ish arrangement. Like the Shi arrangement, the Ish arrangement begins with the $\left(\begin{array}{l}n \\ 2\end{array}\right)$ linear hyperplanes of the Coxeter arrangement and then adds another $\left(\begin{array}{l}n \\ 2\end{array}\right)$ affine hyperplanes:

$$
\operatorname{Ish}(n):=\operatorname{Cox}(n) \cup\left\{e_{i}-e_{n}=a: 1 \leq i \leq n-1, a \in\{1, \ldots, n-i\}\right\} .
$$

Figure 1.2 displays the arrangements Shi(3) and Ish(3). Note that each has 16 chambers and 4 bounded chambers. There is an important reason for this: the arrangements $\operatorname{Shi}(n)$ and $\operatorname{Ish}(n)$ share the same characteristic polynomial, as we now show.

To avoid extra notation, we will use a non-standard definition of the characteristic polynomial. This definition is due to Crapo and Rota, and was applied extensively by Athanasiadis - see Stanley [29, Lecture 5] for details. Let $\mathcal{A}$ be an arrangement of finitely many hyperplanes in $\mathbb{R}^{n}$. Suppose further that each of these hyperplanes has an equation with integer coefficients.
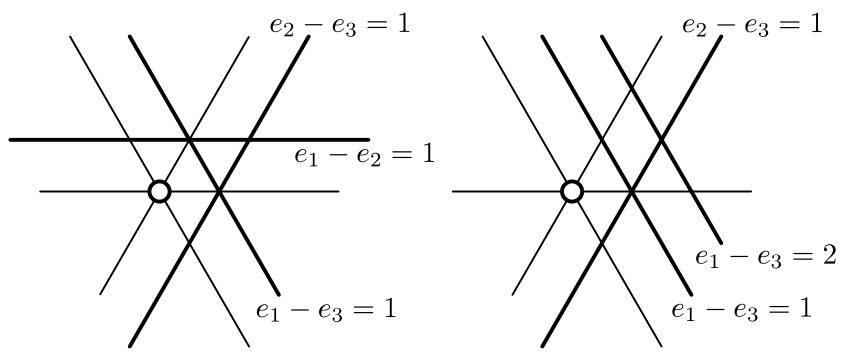

Figure 1.2: The arrangements Shi(3) and Ish(3). 
Then, given a (large) finite field $\mathbb{F}_{q}$ with $q$ elements, we may consider the reduced arrangement $\mathcal{A}_{q}$ in $\mathbb{F}_{q}^{n}$. It turns out that (for all but finitely many $q$ ), the number of points of $\mathbb{F}_{q}^{n}$ not on any hyperplane of $\mathcal{A}_{q}$ is given by a polynomial in $q$, called the characteristic polynomial of $\mathcal{A}$ :

$$
\chi(\mathcal{A}, q)=\#\left(\mathbb{F}_{q}^{n}-\cup_{H \in \mathcal{A}_{q}} H\right)=q^{n}-\# \cup_{H \in \mathcal{A}_{q}} H .
$$

The characteristic polynomial of the Shi arrangement is well known (cf. [29, Theorem 5.16]). Our new result is the following.

Theorem 1.1. The Shi arrangement and the Ish arrangement (in $\mathbb{R}^{n}$ ) share the same characteristic polynomial, viz.

$$
\chi(\operatorname{Ish}(n), q)=q(q-n)^{n-1} .
$$

Proof. Let $p$ be a large prime and consider a regular $p$-gon whose vertices represent the elements of the finite field $\mathbb{F}_{p}=\{1,2, \ldots, p\}$, in clockwise order. We will think of a vector $v=\left(v_{1}, \ldots, v_{n}\right) \in \mathbb{F}_{p}^{n}$ as a labeling of the vertices, as follows: if $v_{i}=k \in \mathbb{F}_{p}$, then place the label $v_{i}$ on the vertex $k$.

To say that $v \in \mathbb{F}_{p}^{n}$ is in the complement of the reduced Ish arrangement $\operatorname{Ish}(n)_{p}$, means that $v_{i}-v_{j} \neq 0$ for all $1 \leq i<j \leq n$ (that is, labels $v_{i}$ and $v_{j}$ do not occupy the same vertex) and $v_{i} \neq v_{n}+a$ for $1 \leq a \leq n-i$ (that is, the label $v_{i}$ does not occur within the $n-i$ vertices clockwise of $v_{n}$ ). To count the vectors in the complement, first note that there are $p$ ways to place the label $v_{n}$. After this, we may place $v_{1}$ in $(p-n)$ ways, since it must avoid the position of $v_{n}$ and the $n-1$ positions just clockwise of this. Next, we may place $v_{2}$ in $(p-n)$ ways since it must avoid the position of $v_{n}$, the $n-2$ positions just clockwise of this, and also the position of $v_{1}$. Continuing in this way, we find that there are $p(p-n)^{n-1}$ vectors in the complement. Since we have established the result for infinitely many $p$, the polynomial is determined.

It is worth noting that the characteristic polynomial is $(q-n)^{n-1}$ when we restrict the arrangements to the hyperplane $\mathbb{R}_{0}^{n}$, because each point of the complement in $\mathbb{R}_{0}^{n}$ corresponds to a line of the complement in $\mathbb{R}^{n}$ (and the line $\mathbb{F}_{q}$ has $q$ points).

The following is a standard result on real hyperplane arrangements. Let $\mathcal{A}$ be an arrangement in a real $d$-dimensional space $V$ and suppose that the normals to $\mathcal{A}$ span a subspace $U \subseteq V$ of dimension $k$ - this $k$ called the rank of $\mathcal{A}$. If $k<d$ then $\mathcal{A}$ has no bounded chambers; its chambers that have a bounded intersection with $U$ are called relatively bounded. 
Zaslavsky's Theorem (see, e.g., Theorem 2.5 of [29]). Let $\mathcal{A}$ be a real arrangement with dimension d and rank $k$. Then:

- The number of chambers of $\mathcal{A}$ is $(-1)^{d} \chi(A,-1)$.

- The number of relatively bounded chambers of $\mathcal{A}$ is $(-1)^{k} \chi(A, 1)$.

If we think of $\operatorname{Shi}(n)$ and $\operatorname{Ish}(n)$ in the space $\mathbb{R}_{0}^{n}$, then $d=k=n-1$.

Corollary 1.2. The arrangements $\operatorname{Shi}(n)$ and $\operatorname{Ish}(n)$ have the same number of chambers - viz. $(n+1)^{n-1}$ - and the same number of bounded chambers - viz. $(n-1)^{n-1}$.

Open Problem. Find a bijective proof of the corollary.

Theorem 1.1 gives a strong sense of "combinatorial symmetry" between the Shi and Ish arrangements. ${ }^{1}$ This observation is at the heart of the paper.

\section{Two statistics on Shi chambers}

Now we define two statistics - called shi and ish - on the chambers of a Shi arrangement (more generally, on the elements of the group $\tilde{\mathfrak{S}}(n)$ ). The first statistic is well known and the second is new. Each statistic counts a certain kind of "inversions" of an affine permutation. We begin by defining these.

\subsection{Affine inversions}

Let $w$ be an element of the (finite) symmetric group $\mathfrak{S}(n)$. If $w(i)>w(j)$ for indices $1 \leq i<j \leq n$ we say that the transposition $(i, j)$ is an inversion of $w$ - equivalently, this means that the hyperplane $e_{i}-e_{j}=0$ separates the cone $w C_{\circ}$ from the dominant cone $C_{\circ}$. The number of inversions of $w$ is called its length.

In the affine symmetric group $\tilde{\mathfrak{S}}(n)$ there is again a correspondence between hyperplanes and transpositions. Recall that the affine transpositions $((i, j))$ and $\left(\left(i^{\prime}, j^{\prime}\right)\right)$ coincide if $i^{\prime}=i+k n$ and $j^{\prime}=j+k n$ for some $k \in \mathbb{Z}$, in which case they both represent the same hyperplane as in (1.2). Hence, each affine transposition has a standard representative in the set

$$
\tilde{T}:=\{((i, j)): 1 \leq i \leq n, i<j\} \subseteq \tilde{\mathfrak{S}}(n) .
$$

Given an affine permutation $\tilde{w} \in \tilde{\mathfrak{S}}(n)$ and an affine transposition $((i, j)) \in$ $\tilde{T}$, we say that $((i, j))$ is an affine inversion of $\tilde{w}$ when $\tilde{w}(i)>\tilde{w}(j)$. Equivalently, the hyperplane (1.2) separates the alcove $\tilde{w} A_{\circ}$ from the fundamental alcove $A_{\circ}$. Again, the (affine) length of $\tilde{w}$ is its number of affine inversions.

\footnotetext{
${ }^{1}$ This combinatorial symmetry has been developed further in [1]. However, the problem of a bijective proof is still open.
} 


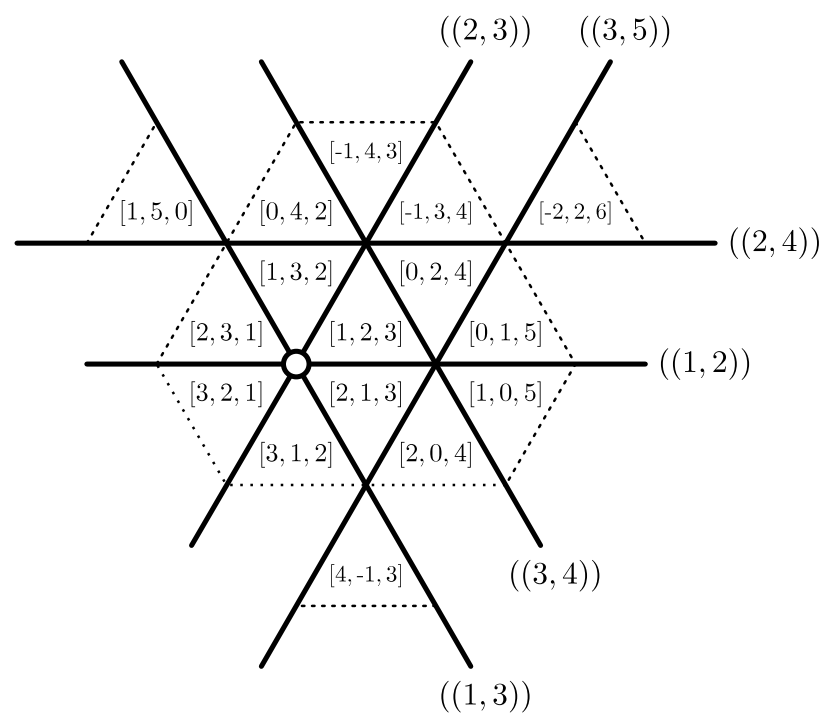

Figure 2.1: Chambers of Shi(3) labeled by affine permutations.

\subsection{The shi statistic}

Each chamber of the Shi arrangement contains a set of alcoves and we will see (Theorem 3.1) that among these is a unique alcove of minimum length - which we call the representing alcove of the chamber, or just a Shi alcove. This defines an injection from Shi chambers into the affine symmetric group. Figure 2.1 displays the representing alcoves for Shi(3), labeled by affine permutations in window notation. We have labeled the Shi hyperplanes with their corresponding affine transpositions,

$$
\operatorname{Shi}(n)=\{((i, j)): 1 \leq i \leq n, i<j<n+i\} .
$$

Definition 2.1. Given a Shi chamber with representing alcove $A$, let $\operatorname{shi}(A)$ denote the number of Shi hyperplanes separating $A$ from the fundamental alcove $A_{\circ}$. Equivalently, if $A=\tilde{w} A_{\circ}$ for affine permutation $\tilde{w} \in \mathfrak{S}(n)$, then $\operatorname{shi}(\tilde{w})$ is the number of affine inversions $((i, j))$ of $\tilde{w}$ satisfying $i<j<n+i$.

For example, consider the permutation $\tilde{w}=[1,5,0]$ in the figure. The inversions of $\tilde{w}$ are $((1,3)),((2,3)),((2,4)),((2,6))$, and hence $\tilde{w}$ has length 4. However, only three of these - viz. $((1,3)),((2,3)),((2,4))$ - come from Shi hyperplanes, hence $\operatorname{shi}(\tilde{w})=3$. 


\subsection{The ish statistic}

To give a natural definition for our second statistic, we must discuss the coset space $\tilde{\mathfrak{S}}(n) / \mathfrak{S}(n)$. By abuse of notation, let $\mathfrak{S}(n)$ denote the subgroup of $\tilde{\mathfrak{S}}(n)$ generated by the subset

$$
I=\left\{s_{1}, \ldots, s_{n-1}\right\} \subseteq\left\{s_{1}, \ldots, s_{n-1}, s_{n}\right\}=S .
$$

In the language of Coxeter groups we say that $\mathfrak{S}(n)$ is a parabolic subgroup of $\tilde{\mathfrak{S}}(n)$. The standard notation for this is to write $\mathfrak{S}(n)=W_{I}$ where $W=$ $\tilde{\mathfrak{S}}(n)$. Then each affine permutation $\tilde{w}$ has a canonical decomposition

$$
\tilde{w}=w_{I} \tilde{w}^{I},
$$

where $w_{I} \in W_{I}$ is a finite permutation and $\tilde{w}^{I} \in W$ is the unique right coset representative of minimum (affine) length. Combinatorially (in window notation), $\left[\tilde{w}^{I}(1), \ldots, \tilde{w}^{I}(n)\right]$ is the increasing rearrangement of $[\tilde{w}(1), \ldots, \tilde{w}(n)]$ and $w_{I}$ is the finite permutation needed to achieve the rearrangement. Geometrically, alcoves of the form $A=\tilde{w}^{I} A_{\circ}$ are precisely those contained in the dominant cone $C_{\circ}$; hence $\tilde{w} A_{\circ}=w_{I} A$ is contained in the cone $w_{I} C_{\circ}$.

We define the ish statistic in terms of minimal coset representatives.

Definition 2.2. Consider a Shi chamber with representing alcove $A$ and suppose that $A=\tilde{w} A_{\circ}$. Its minimal coset representative $\tilde{w}^{I} A_{\circ}$ is the corresponding alcove in the dominant cone $C_{\circ}$. Let ish $(A)$ denote the number of hyperplanes of the form $e_{i}-e_{n}=a$ (with $1 \leq i \leq n-1$ and $a \in \mathbb{Z}$ ) separating $\tilde{w}^{I} A_{\circ}$ from the fundamental alcove $A_{\circ}$. Equivalently, let ish $(\tilde{w})$ denote the number of affine inversions of $\tilde{w}^{I}$ of the form $((n, j))$ with $n<j$.

Two notes: In order to facilitate later generalization, we have defined ish in terms of all hyperplanes of the form $e_{i}-e_{n}=a$. In our current context, however, only the Ish hyperplanes (i.e. $a \in\{1, \ldots, n-i\}$ ) will contribute. We also emphasize the fact that ish is a statistic on the (representing alcoves of) Shi chambers, not on the Ish chambers. It seems that the chambers of the Ish arrangement are not so natural.

For example, consider the affine permutation $\tilde{w}=[-1,4,3]$, as shown in Figure 2.1. It is contained in the cone $[1,3,2] C_{\circ}$ and its increasing rearrangement is $[-1,3,4]$. Hence, it has parabolic decomposition

$$
[-1,4,3]=\tilde{w}=w_{I} \tilde{w}^{I}=[1,3,2][-1,3,4] .
$$




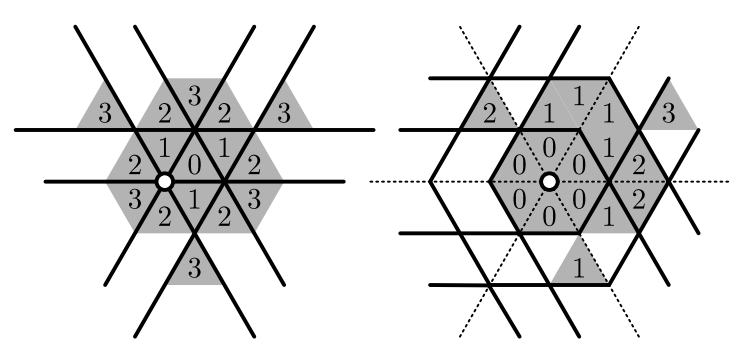

Figure 2.2: The shi and ish statistics on the chambers of Shi(3).

The inversions of $\tilde{w}^{I}=[-1,3,4]$ are $((2,4))$ and $((3,4))$, of which only the second is an Ish hyperplane; hence ish $(\tilde{w})=1$. In Figure 2.2 we have displayed the shi and ish statistics for all chambers of Shi(3). (Note: to compute ish by hand, one may extend the Ish hyperplanes from the dominant cone to the other cones by reflection.) Their joint-distribution is recorded in the following table:

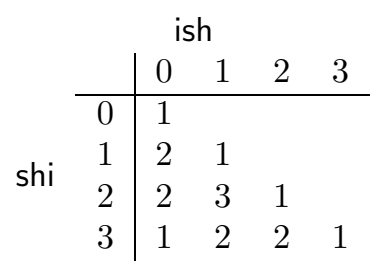

\subsection{Theorems and a conjecture}

We will make four assertions and then describe our state of knowledge about them (i.e. whether each is a Theorem or a Conjecture). We will use the following notation.

Recall from (1.1) that $\mathcal{D H}(n ; q, t)$ denotes the bigraded Hilbert series of the ring of diagonal harmonic polynomials. Define

$$
\operatorname{Shi}(n ; q, t):=\sum_{A} q^{\mathrm{ish}(A)} t_{\left(\begin{array}{c}
n \\
2
\end{array}\right)-\operatorname{shi}(A)},
$$

where the sum is taken over representing alcoves $A$ for all of the chambers of the arrangement $\operatorname{Shi}(n)$. We say that an alcove is positive if it is contained in the dominant cone $C_{\circ}$ (i.e. if $A$ is on the "positive" side of each generating hyperplane for $\mathfrak{S}(n))$. Let $\mathrm{Shi}_{+}(n ; q, t)$ denote the corresponding sum over positive Shi alcoves. Finally, consider the standard $q$-integer, $q$-factorial, and $q$-binomial coefficient: 


$$
\begin{aligned}
{[a]_{q} } & =1+q+\cdots+q^{a-1} \\
{[a]_{q} ! } & =[a]_{q}[a-1]_{q} \cdots[2]_{q}[1]_{q} \\
{\left[\begin{array}{l}
a \\
b
\end{array}\right]_{q} } & =\frac{[a]_{q} !}{[a-b]_{q} ![b]_{q} !}
\end{aligned}
$$

\section{Assertions.}

(1) $\operatorname{Shi}(n ; q, t)=\mathcal{D H}(n ; q, t)$, and hence is symmetric in $q$ and $t$.

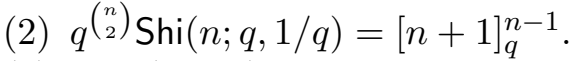

(3) $\mathrm{Shi}_{+}(n ; q, t)$ is equal to Garsia and Haiman's $q, t$-Catalan number, and hence is symmetric in $q$ and $t$.

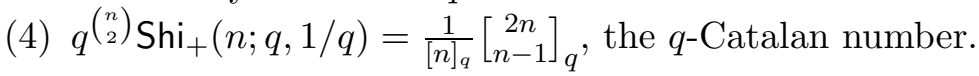

In particular, note that $q^{\left(\begin{array}{c}n \\ 2\end{array}\right)} \mathrm{Shi}_{+}(n ; q, 1 / q)$ is equal to the sum of $q^{\text {shi }(A)+i s h(A)}$ over the positive Shi alcoves $A$. For $n=3$ we may compute this sum using the data in Figure 2.2 to obtain

$$
1+q^{2}+q^{3}+q^{4}+q^{6}=\frac{[6]_{q}[5]_{q}}{[3]_{q}[2]_{q}}=\frac{1}{[3]_{q}}\left[\begin{array}{l}
6 \\
2
\end{array}\right]_{q},
$$

which is a $q$-Catalan number. One may check that the other three assertions are also true in the case $n=3$.

In the following section we will establish a bijection (Main Theorem 3.6) from Shi chambers to labeled lattice paths, which sends our statistics (ish, shi$\left(\begin{array}{l}n \\ 2\end{array}\right)$ ) to the statistics (bounce, area') of Haglund and Loehr [16]. This allows us to clarify the Assertions.

Status. Each of the following results depends on our Main Theorem 3.6.

(1) Conjecture. This is equivalent to a conjecture of Haglund and Loehr [16] (known in a different form to Haiman). No combinatorial explanation of the $q, t$ symmetry is known.

(2) Theorem. This is equivalent to a theorem of Loehr [17].

(3) Theorem. This follows from theorems of Garsia and Haglund $[9,10]$. No combinatorial explanation of the $q, t$ symmetry is known.

(4) Theorem. This is equivalent to a theorem of Haglund [14], which was later proved bijectively by Loehr [18].

\section{Shi chambers and lattice paths}

In this section we will prove the above stated results regarding the shi and ish statistics. To do this we interpret Shi chambers as certain labeled lattice paths. 


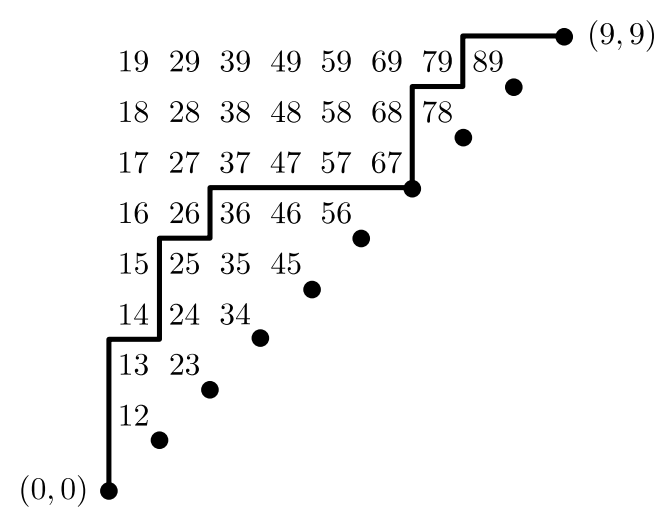

Figure 3.1: An ideal and its corresponding Dyck path.

\subsection{The root poset and Dyck paths}

Cartan and Killing invented root systems prior to 1890 and used these to classify the semisimple Lie algebras. In this paper we are primarily concerned with the "type $A$ " root system, which is related to the symmetric group. Recall that $\mathfrak{S}(n)$ has a faithful action on $\mathbb{R}_{0}^{n}$ generated by the reflections $S=$ $\left\{s_{1}, s_{2}, \ldots, s_{n-1}\right\}$, where $s_{i}$ is the reflection in the hyperplane $e_{i}-e_{i+1}=0$. The positive normal vectors to the generating hyperplanes form a special basis, called the basis of simple roots $\Delta=\left\{e_{1}-e_{2}, \ldots, e_{n-1}-e_{n}\right\}$. The positive normal vectors to all reflecting hyperplanes form the set of positive roots $\Phi^{+}=\left\{e_{i}-e_{j}: 1 \leq i<j \leq n\right\}$.

The root poset is a partial order on $\Phi^{+}$defined as follows. Given two positive roots $\alpha, \beta \in \Phi^{+}$we say that $\alpha \leq \beta$ whenever $\beta-\alpha$ can be written in the basis $\Delta$ using non-negative coefficients. Equivalently, we have $\alpha \leq \beta$ when $\beta-\alpha$ is in the positive cone generated by $\Delta$. In type $A$ this means that $e_{j}-e_{k} \leq e_{i}-e_{\ell}$ if and only if $i \leq j<k \leq \ell$.

In this paper we will visualize the root poset in a particular way. Consider an array of integer points $(i, j), 1 \leq i<j \leq n$, and place the label " $i j$ " in the unit square with top right corner $(i, j)$. (See Figure 3.1.) This square will represent the root $e_{i}-e_{j}$. Thus for $\alpha, \beta \in \Phi^{+}$we have $\alpha \leq \beta$ when the square labeled $\beta$ occurs weakly to the left and weakly above the square labeled $\alpha$.

A set of roots $\mathcal{I} \subseteq \Phi^{+}$is called an ideal if $\alpha \in \mathcal{I}$ and $\alpha \leq \beta$ together imply $\beta \in \mathcal{I}$. We may picture this as a collection of unit squares aligned up and to the left. The lower boundary of these squares defines a lattice path from $(0,0)$ to $(n, n)$ which 
- uses only steps of the form $(0,1)$ and $(1,0)$, and

- stays weakly above the diagonal.

This defines a bijection between ideals in $\Phi^{+}$and so-called Dyck paths. For example, Figure 3.1 displays an ideal in the root poset of $\mathfrak{S}(9)$ and its corresponding Dyck path.

\subsection{Shi alcoves}

3.2.1. The address of an alcove For each root $\alpha \in \Phi^{+}$and each real number $k \in \mathbb{R}$ let $H_{\alpha, k}$ denote the hyperplane $\left\{x \in \mathbb{R}_{0}^{n}:(x, \alpha)=k\right\}$. When $\alpha=e_{i}-e_{j}$ this is the hyperplane $e_{i}-e_{j}=k$ (in the language of Section 1.2). Now let $A$ be an alcove of the affine arrangement. For each root $\alpha \in \Phi^{+}$there exists a unique integer $k_{A}(\alpha)$ such that $A$ lies between the hyperplanes $H_{\alpha, k_{A}(\alpha)}$ and $H_{\alpha, k_{A}(\alpha)+1}$. The function $k_{A}: \Phi^{+} \rightarrow \mathbb{Z}$ determines the position of $A$, so we call it the address of $A$. An important result of J.-Y. Shi characterizes which functions can be addresses (see Sommers [27, Proposition 4.1], which is a restatement of J.-Y. Shi [24, Theorem 5.2]).

Shi's Theorem. A function $k: \Phi^{+} \rightarrow \mathbb{Z}$ is the address of an alcove if and only if, for all triples $\alpha, \beta, \alpha+\beta$ of positive roots, we have

$$
k(\alpha)+k(\beta) \leq k(\alpha+\beta) \leq k(\alpha)+k(\beta)+1 .
$$

We say that the alcove $A$ is positive if it lies in the dominant cone $C_{0}$. Equivalently, $A$ is positive if and only if its address $k_{A}$ takes non-negative values. We observe that the address of a positive alcove is an increasing function on the root poset. Indeed, if $\alpha \leq \beta$ then $\beta-\alpha$ is a non-negative integer combination of simple roots. Morever, there exists a way to get from $\alpha$ to $\beta$ by successively adding these simple roots, always staying within $\Phi^{+}$. Since we assumed that $k_{A}(\gamma) \geq 0$ for all simple $\gamma \in \Delta \subseteq \Phi^{+}$, the result follows from Shi's Theorem.

3.2.2. Positive Shi alcoves The Shi arrangement consists of the hyperplanes $H_{\alpha, k}$ for all $\alpha \in \Phi^{+}$and $k \in\{0,1\}$. Given an alcove $A$, we would like to understand in which chamber of the Shi arrangement it lies. This problem is easiest to solve for positive alcoves; in this case we need only specify for which roots $k_{A}$ is zero and for which roots it is positive. To this end, we define

$$
\mathcal{I}_{A}:=k_{A}^{-1}(\{1,2, \ldots\}) \subseteq \Phi^{+} .
$$




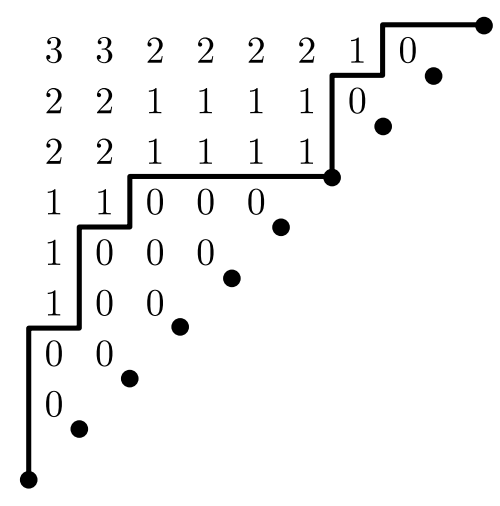

Figure 3.2: The address of a positive Shi alcove.

Since the address of a positive alcove $A$ is increasing, we observe in this case that $\mathcal{I}_{A} \subseteq \Phi^{+}$is an ideal in the root poset. It turns out that this defines a bijection between positive Shi chambers and ideals. For this result we refer to Sommers [27, Lemmas 5.1 and 5.2].

Theorem 3.1 (Representing Alcoves). Given an ideal $\mathcal{I} \subseteq \Phi^{+}$of positive roots, there exists a unique positive alcove of minimum length such that $\mathcal{I}=\mathcal{I}_{A}$. The address of this alcove is given by $k_{\mathcal{I}}: \Phi^{+} \rightarrow \mathbb{Z}$ where $k_{\mathcal{I}}(\alpha)$ is the maximum number $r$ such that $\alpha$ can be expressed as a sum of $r$ roots in the ideal $\mathcal{I}$.

We call the unique minimum alcove in a positive Shi chamber its representing alcove, or just a positive Shi alcove. Figure 3.2 displays the address of the representing alcove corresponding to the ideal in Figure 3.1.

3.2.3. Non-positive Shi alcoves It is true that each non-positive Shi chamber also contains a unique alcove of minimum length, which we call a non-positive Shi alcove. Unfortunately, we do not know an expression for the address of such an alcove in the spirit of Theorem 3.1. Instead we use a slightly weaker result due to Pak and Stanley (see [28, Theorem 5.1]).

Recall that a positive Shi chamber $C$ corresponds to an ideal $\mathcal{I} \subseteq \Phi^{+}$of positive roots: given a positive root $\alpha=e_{i}-e_{j}$, the chamber $C$ lies on the positive side of $H_{\alpha, 1}$ when $\alpha \in \mathcal{I}$ and $C$ lies between $H_{\alpha, 0}$ and $H_{\alpha, 1}$ when $\alpha \notin \mathcal{I}$. In addition, the minimal roots $\alpha \in \mathcal{I}$ (i.e. such that $\mathcal{I}-\alpha$ is also an ideal) correspond exactly to the hyperplanes $H_{\alpha, 1}$ that support a facet of the chamber and also separate it from the fundamental alcove $A_{\circ}$. We call these the floors of the chamber. In the language of Dyck paths, these are the 
squares contained in the "valleys" of the path. For instance, the valleys in Figure 3.1 contain roots $e_{1}-e_{4}, e_{2}-e_{6}, e_{6}-e_{7}$ and $e_{7}-e_{9}$.

Now consider a non-positive Coxeter cone $w C_{\circ}$, with $w \in \mathfrak{S}(n)$. The Shi hyperplanes that intersect the dominant cone $C_{\circ}$ are precisely $e_{i}-e_{j}=1$ for $1 \leq i<j \leq n$. Let us say that the transposition $(i, j)$ with $i<j$ is a non-inversion of $w$ if $w(i)<w(j)$. Then the Shi hyperplanes intersecting $w C_{\circ}$ biject to non-inversions of $w$ as follows. Note that we have $i<j$ if and only if the hyperplane $e_{i}-e_{j}=1$ intersects $C_{\circ}$ if and only if the hyperplane $e_{w(i)}-e_{w(j)}=1$ intersects $w C_{\circ}$. Furthermore, the hyperplane $e_{w(i)}-e_{w(j)}=1$ occurs in the Shi arrangement if and only if $w(i)<w(j)$. Thus the Shi hyperplanes intersecting the cone $w C_{\circ}$ are precisely $e_{w(i)}-e_{w(j)}=1$, where $i<j$ and $w(i)<w(j)$.

Finally, let $A$ be a minimal alcove for some Shi chamber in $w C_{\circ}$. Then $w^{-1} A$ is a Shi alcove in $C_{\circ}$ and the floors of $A$ biject to the floors of $w^{-1} A$ via $w^{-1}$. In other words, the floors of $A$ are of the form $e_{w(i)}-e_{w(j)}=1$ where $w(i)<w(j)$ and the label " $i j$ " occurs in a valley of the Dyck path corresponding to $w^{-1} A \subseteq C_{\circ}$. In summary, we have the following.

Theorem 3.2 (Pak and Stanley [28]). The chambers of the Shi arrangement are in bijection with pairs $(w, \mathcal{I})$ where $w \in \mathfrak{S}(n)$ is a permutation and $\mathcal{I} \subseteq \Phi^{+}$is an ideal of positive roots (a Dyck path) such that the minimal elements of $\mathcal{I}$ (labels in the valleys of the path) are a subset of non-inversions of $w$.

Figure 3.3 shows an example corresponding to the permutation

$$
w=521863497 \in \mathfrak{S}(9)
$$

and the same path $\mathcal{I}$ as in Figures 3.1 and 3.2. This diagram corresponds to the non-positive Shi alcove $w A$, where $A$ is the positive Shi alcove corresponding to $\mathcal{I}$. Here the symbols $\times$ and $\bigcirc$ represent, respectively, inversions and non-inversions of $w$. The fact that the valleys of $\mathcal{I}$ contain $\bigcirc$ 's means precisely that $w A$ is a minimal alcove in some Shi chamber. We call such a diagram a labeled Dyck path.

Let us interpret the statistics shi and ish in terms of labeled Dyck paths.

3.2.4. $\left(\begin{array}{c}\boldsymbol{n} \\ 2\end{array}\right)-\mathbf{s h i}=$ area $^{\prime} \quad$ In [16] Haglund and Loehr defined two statistics on labeled Dyck paths - called area' and bounce - and they conjectured that the generating function $\sum q^{\text {area' }} t^{\text {bounce }}$ equals the bigraded Hilbert series $\mathcal{D H}(n ; q, t)$ of diagonal harmonic polynomials.

We first deal with area', which Haglund and Loehr defined as the number of non-inversions of $w$ below the labeled Dyck path $(w, \mathcal{I})$. When 


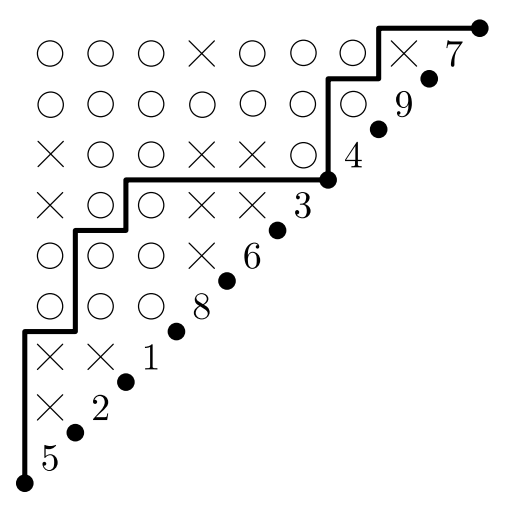

Figure 3.3: A non-positive Shi chamber.

$w$ is the identity permutation, this is just the number of unit squares fully between the path and the diagonal, i.e. the "area" of the path.

Theorem 3.3. Let $A=\tilde{w} A_{\circ}$ be a (positive or non-positive) Shi alcove, where $w=w_{I} \in \mathfrak{S}(n)$ is the finite permutation corresponding to $\tilde{w}=w_{I} \tilde{w}^{I} \in$ $\tilde{\mathfrak{S}}(n)$, and $(w, \mathcal{I})$ is the corresponding labeled Dyck path. Then we have

$$
\left(\begin{array}{l}
n \\
2
\end{array}\right)-\operatorname{shi}(A)=\operatorname{area}^{\prime}(w, \mathcal{I}) \text {. }
$$

Proof. Recall that shi $(A)$ is the number of Shi hyperplanes separating $A$ from the fundamental alcove $A_{\circ}$. These come in two classes. First, it is well known that the hyperplanes separating $A_{\circ}$ from $w A_{\circ}$ are exactly $e_{i}-e_{j}=0$ such that $1 \leq i<j \leq n$ and $w(i)>w(j)$; i.e. the inversions of $w$. These are all of the $\times$ 's in the diagram. Second, the hyperplanes of the form $e_{i}-e_{j}=1$ separating $A=\tilde{w} A_{\circ}$ from $w A_{\circ}$ correspond to the cells above the path. Such a hyperplane is a Shi hyperplane precisely when $w(i)<w(j)$, so these correspond to the $\bigcirc$ 's above the path. Finally, since the total number of symbols is $\left(\begin{array}{l}n \\ 2\end{array}\right)$ we conclude that $\left(\begin{array}{l}n \\ 2\end{array}\right)-\operatorname{shi}(A)$ is the number of $\bigcirc$ 's below the path.

3.2.5. ish $=$ bounce The bounce statistic was discovered by Haglund in 2003 [14]. It provided the first combinatorial interpretation of the $q, t$ Catalan numbers of Garsia and Haiman. Haglund and Loehr [16] later extended the statistic to labeled Dyck paths $(w, \mathcal{I})$ by defining bounce $(w, \mathcal{I})=$ bounce $(\mathcal{I})$ (i.e. ignoring the labeling by $w$ ). 


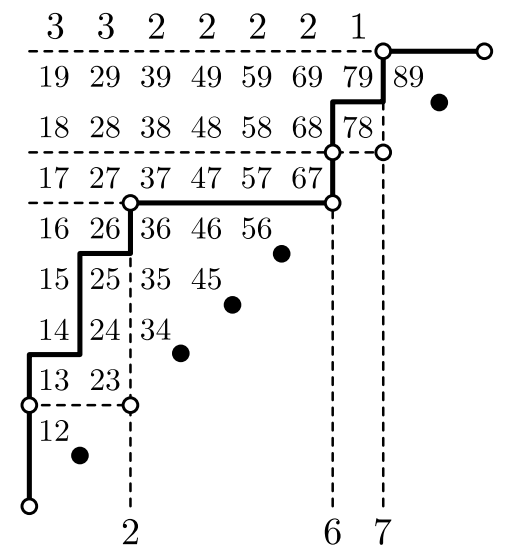

Figure 3.4: The bounce path decomposition.

Definition 3.4 (Haglund). Given a Dyck path $\mathcal{I}$, we construct its bounce path as follows. Begin at $(n, n)$ and travel left until traveling further left would cross the path $\mathcal{I}$, then travel down until we hit the diagonal. Repeat these two steps until we hit $(0,0)$. Define bounce $(\mathcal{I})$ as the sum of $i$ between 1 and $n-1$ such that the bounce path contains the diagonal point $(i, i)$.

For example, let $\mathcal{I}$ be the Dyck path (given by the solid lines) in Figure 3.4. Here the bounce path is indicated by the white vertices, and the numbers along the bottom show that bounce for this path is

$$
\operatorname{bounce}(\mathcal{I})=7+6+2=15 \text {. }
$$

Now let us compute ish for this example. Recall that the Dyck path $\mathcal{I}$ corresponds to a positive Shi alcove $A=\tilde{w} A_{\circ}$ with address $k_{\mathcal{I}}: \Phi^{+} \rightarrow \mathbb{Z}$ given by Figure 3.2. Since $A$ is positive, we know that $\tilde{w}$ has minimum length in the right coset $\mathfrak{S}(n) \tilde{w}$. Hence ish $(A)$ is defined as the number of hyperplanes of the form $e_{i}-e_{n}=a$ (for some $a \in \mathbb{Z}$ ) separating $A$ from the fundamental alcove $A_{\circ}$. By the definition of the address $k_{\mathcal{I}}$, this is just the sum of the numbers in the top row of Figure 3.2; i.e.

$$
\operatorname{ish}(\mathcal{I})=3+3+2+2+2+2+1=15 .
$$

As we will see, it is not a coincidence that $(7,6,2)$ and $(3,3,2,2,2,2,1)$ are conjugate partitions of the integer 15 . 
Theorem 3.5. Given a (positive or non-positive) Shi alcove $A$ and its corresponding labeled Dyck path $(w, \mathcal{I})$ we have

$$
\operatorname{ish}(A)=\operatorname{bounce}(w, \mathcal{I}):=\operatorname{bounce}(\mathcal{I})
$$

Proof. Suppose that $A=\tilde{w} A_{\circ}$ where $\tilde{w}$ is an affine permutation $\tilde{w} \in \tilde{\mathfrak{S}}(n)$. Suppose further that $\tilde{w}=w_{I} \tilde{w}^{I}$ where $w_{I} \in \mathfrak{S}(n) \subseteq \tilde{\mathfrak{S}}(n)$ is a finite permutation and $\tilde{w}^{I}$ is the minimal coset representative. The alcove $A$ thus corresponds to a labeled Dyck path $\left(w_{I}, \mathcal{I}\right)$ and the positive alcove $\tilde{w}^{I} A_{\circ}$ corresponds to the "unlabeled" Dyck path $(1, \mathcal{I})=\mathcal{I}$.

Recall that ish $(A)$ is the number of hyperplanes between $\tilde{w}^{I} A_{\circ}$ and $A_{\circ}$ of the form $e_{i}-e_{n}=a$ (for $a \in \mathbb{Z}$ ). Given $\alpha=e_{i}-e_{n}$ the number of these hyperplanes is exactly $k_{A}(\alpha)$, where $k_{A}: \Phi^{+} \rightarrow \mathbb{Z}$ is the address of the positive Shi alcove $\tilde{w}^{I} A_{\circ}$. Thus ish $(A)=\operatorname{ish}(\mathcal{I})$ is the $\operatorname{sum} \sum_{i=1}^{n-1} k_{A}(i n)$ where in denotes a cell in the top row of our lattice path picture.

By Theorem 3.1, we know that $k_{A}(\alpha)=k_{\mathcal{I}}(\alpha)$ equals the maximum integer $r$ such that $\alpha$ can be written as a sum of $r$ roots above the Dyck path $\mathcal{I}$. Now consider the bounce path of $\mathcal{I}$ and extend it to the left from each point at which it hits $\mathcal{I}$ (as we have done in Figure 3.4). This decomposes the cells above $\mathcal{I}$ into "blocks" (there are 3 blocks in Figure 3.4). Let the blocks be denoted $B_{1}, B_{2}, \ldots, B_{m}$ from lowest to highest and let $\ell\left(B_{j}\right)$ denote the number of cells in the top row of block $B_{j}$. By construction we see that the bounce path of $\mathcal{I}$ hits the diagonal precisely at the points $\left(\ell\left(B_{j}\right), \ell\left(B_{j}\right)\right)$ for $j \in\{1, \ldots, m\}$, hence bounce $(\mathcal{I})=\sum_{j=1}^{m} \ell\left(B_{j}\right)$ equals the number of cells in the top rows of the blocks.

On the other hand, we could count these cells by columns. Note that the number of such cells in column $i$ equals the number of blocks that intersect column $i$. If we can show that this number equals $k_{A}(i n)$, we will have proved that

$$
\operatorname{ish}(\mathcal{I})=\sum_{i=1}^{n-1} k_{\mathcal{I}}(\text { in })=\sum_{j=1}^{m} \ell\left(B_{j}\right)=\operatorname{bounce}(\mathcal{I}) .
$$

So consider the root $\alpha=e_{i}-e_{n}$ corresponding to cell $i n$ and suppose we have $\alpha=\gamma_{1}+\cdots+\gamma_{r}$ where each $\gamma_{i}$ is a root above the Dyck path $\mathcal{I}$. By [27, Lemma 3.2], we can reorder the summands such that $\gamma_{i}=e_{b_{i}}-e_{b_{i+1}}$ for some $1 \leq b_{1}<b_{2}<\cdots<b_{r+1} \leq n$. (In Figure 3.5 we have shown an example where $\alpha=e_{1}-e_{9}$ and $\left(b_{1}, b_{2}, b_{3}, b_{4}\right)=(1,6,7,9)$.) In this case $\gamma_{i+1}$ is above and to the right of $\gamma_{i}$ and one gets from $\gamma_{i}$ to $\gamma_{i+1}$ by "bouncing" off the diagonal. By construction, this means that $\gamma_{i}$ and $\gamma_{i+1}$ occur in distinct blocks; hence $\gamma_{1}, \ldots, \gamma_{r}$ occur in $r$ distinct blocks, all of which intersect 


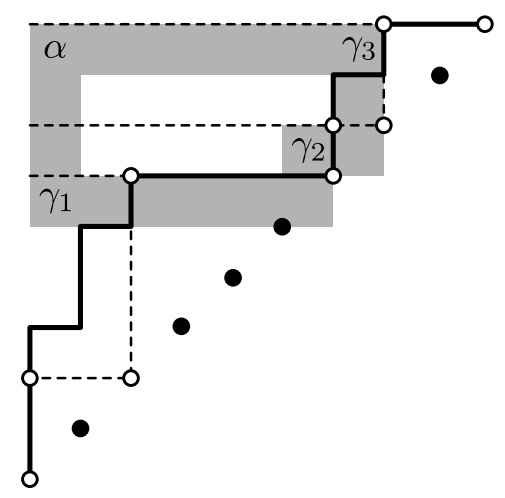

Figure 3.5: The root $\alpha$ as a sum of three roots $\gamma_{1}+\gamma_{2}+\gamma_{3}$ above the path.

column $i$. Hence the maximum $r$ for which this is possible (which equals $k_{\mathcal{I}}($ in $)$ by Theorem 3.1) is at most the number of blocks that intersect column $i$.

We claim that in fact $k_{\mathcal{I}}(\alpha)$ (for $\alpha=e_{i}-e_{n}$ ) equals the number of blocks (say $m$ ) that intersect column $i$. We will show this by finding roots $\gamma_{1}, \ldots, \gamma_{m}$ above the path (i.e. one from each block intersecting column $i$ ) such that $\alpha=\gamma_{1}+\cdots+\gamma_{m}$. To begin, choose $\gamma_{1}$ in column $i$ to correspond to the highest cell in the lowest block that intersects this column. Now travel to the right from $\gamma_{1}$, bounce off the diagonal, and travel up until we reach a cell $\gamma_{2}$ such that: $\gamma_{2}$ is in the block just above the block containing $\gamma_{1}$, and $\gamma_{2}$ is in the highest row of this block. This is always possible. (See Figure 3.5; we are really just following the bounce path.) Continue in this way to achieve the desired decomposition $\alpha=\gamma_{1}+\cdots+\gamma_{m}$.

In conclusion, here is the main result of the paper.

Main Theorem 3.6. The bijection $A \mapsto(w, \mathcal{I})$ from Shi alcoves to labeled Dyck paths sends the pair of statistics $\left(\left(\begin{array}{l}n \\ 2\end{array}\right)-\right.$ shi, ish) to the pair (area', bounce).

\section{The inverse statistics}

We chose the definitions of shi and ish to emphasize their connection with the Ish hyperplane arrangement. However, we will obtain a more natural interpretation of ish when we compose it with inversion in the affine Weyl group. That is, let us define the following inverse statistics. 
Definition 4.1. For any affine permutation $\tilde{w} \in \tilde{\mathfrak{S}}(n)$, we define

$$
\begin{aligned}
& \operatorname{shi}^{-1}(\tilde{w}):=\operatorname{shi}\left(\tilde{w}^{-1}\right) \quad \text { and } \\
& \operatorname{sish}^{-1}(\tilde{w}):=\operatorname{ish}\left(\tilde{w}^{-1}\right) .
\end{aligned}
$$

First let us say why we care about the inverse statistics.

\subsection{Inverse Shi alcoves}

Let $E$ denote the set of representing alcoves for the chambers of the Shi arrangement Shi $(n)$ (see Theorem 3.1). Thinking of these alcoves as elements of the affine symmetric group $\tilde{\mathfrak{S}}(n)$, we may invert them. J.-Y. Shi showed that the set $E^{-1}$ of inverted alcoves has a remarkable shape (see [25]).

Theorem 4.2. The inverted Shi alcoves $E^{-1}$ are precisely the alcoves inside the simplex $D^{n+1}(n) \subseteq \mathbb{R}_{0}^{n}$ bounded by the hyperplanes

$$
\left\{e_{i}-e_{i+1}=-1: 1 \leq i \leq n-1\right\} \cup\left\{e_{1}-e_{n}=2\right\},
$$

which is congruent to the dilation $(n+1) A_{\circ}$ of the fundamental alcove $A_{\circ}$.

Since the dimension of the space $\mathbb{R}_{0}^{n}$ is $n-1$, the simplex $D^{n+1}(n)$ contains $(n+1)^{n-1}$ alcoves. Shi concluded that his arrangement has $(n+1)^{n-1}$ chambers. Figure 4.1 displays the simplex $D^{4}(3)$ and the Shi arrangement in $\mathbb{R}_{0}^{3}$. We have labeled each alcove by the window notation for the inverse of the corresponding affine permutation. Compare to Figure 2.1.

Following Main Theorem 3.6, we assert that the joint-distribution of shi ${ }^{-1}$ and ish ${ }^{-1}$ on the simplex $D^{n+1}(n)$ is the bigraded Hilbert series of diagonal harmonic polynomials. In fact, since the shape $D^{n+1}(n)$ (Figure 4.1) is much nicer than the distribution of Shi alcoves (Figure 2.1), it seems that the inverse statistics shi $^{-1}$ and ish ${ }^{-1}$ are more important than the originals. Thus we would like to understand them directly, without reference to inversion in $\tilde{\mathfrak{S}}(n)$.

\subsection{The inverse shi statistic}

To do this we need to discuss the realization of the affine symmetric group $\tilde{\mathfrak{S}}(n)$ as a semi-direct product of the finite symmetric group $\mathfrak{S}(n)$ and the root lattice

$$
Q=\left\{(r(1), \ldots, r(1)) \in \mathbb{Z}^{n}: r(1)+\cdots+r(n)=0\right\} .
$$




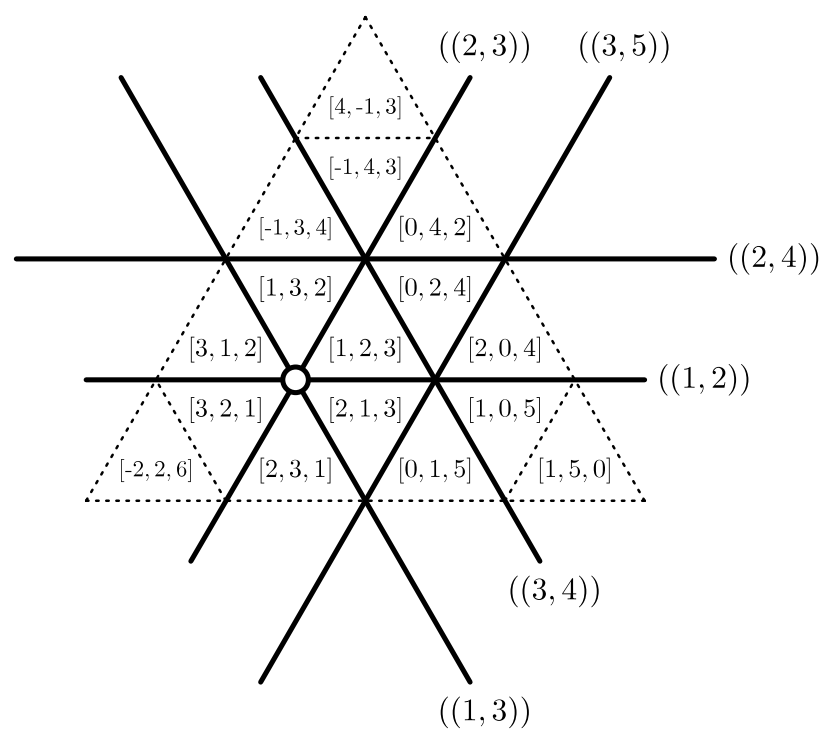

Figure 4.1: The simplex $D^{4}(3)$ of inverted Shi alcoves.

By abuse of notation, we think of $Q$ as an abelian group by associating the root $r \in Q$ with the translation $t_{r}: \mathbb{R}_{0}^{n} \rightarrow \mathbb{R}_{0}^{n}$ defined by $t_{r}(v)=v+r$. Then $\tilde{\mathfrak{S}}(n)$ is the semi-direct product $Q \rtimes \mathfrak{S}(n)=\left\{t_{r} w: x \in \mathfrak{S}(n), r \in Q\right\}$, where $w \in \mathfrak{S}(n)$ acts on $t_{r} \in Q$ by $w t_{r} w^{-1}=t_{w(r)}$. Note in particular that inversion is given by $\left(t_{r} w\right)^{-1}=t_{-w^{-1}(r)} w^{-1}$.

The semi-direct product structure $\tilde{\mathfrak{S}}(n)=Q \rtimes \mathfrak{S}(n)$ has the following combinatorial interpretation in terms of the window notation. Recall that an affine permutation $\tilde{w}: \mathbb{Z} \rightarrow \mathbb{Z}$ must satisfy $\tilde{w}(k+n)=\tilde{w}(k)+n$ for all $k \in \mathbb{Z}$, and $\tilde{w}(1)+\cdots+\tilde{w}(n)=\left(\begin{array}{c}n+1 \\ 2\end{array}\right)$. If we denote $\tilde{w} \in \tilde{\mathfrak{S}}(n)$ by the vector $\tilde{w}=[\tilde{w}(1), \ldots, \tilde{w}(n)]$, then each affine permutation has a unique decomposition,

$$
[\tilde{w}(1), \ldots, \tilde{w}(n)]=(w(1), \ldots, w(n))+n(r(1), \ldots, r(n))
$$

where $w \in \mathfrak{S}(n)$ is a finite permutation and $r=(r(1), \ldots, r(n))$ is an element of the root lattice $Q$. For example, the affine permutation $[-2,2,6] \in \tilde{\mathfrak{S}}(3)$ decomposes as

$$
[-2,2,6]=(1,2,3)+3(-1,0,1) .
$$

One may easily check that the map $\tilde{w}=w+n r \leftrightarrow \tilde{w}=t_{r} w$ is an isomorphism between the two structures. 
We can now describe the inverse shi statistic explicitly. In fact, it doesn't change.

Theorem 4.3. For any affine permutation $\tilde{w} \in \tilde{\mathfrak{S}}(n)$ we have

$$
\operatorname{shi}^{-1}(\tilde{w})=\operatorname{shi}(\tilde{w})
$$

Proof. First recall that the Shi arrangement Shi $(n)$ consists of all the affine hyperplanes $H_{\alpha, k}$ that touch the closure of the fundamental alcove $A_{\circ}{ }^{2}$ There are two of these perpendicular to each root $\alpha \in \Phi^{+}$; namely $H_{\alpha, 0}$ and $H_{\alpha, 1}$.

The inversions of $\tilde{w} \in \mathfrak{S}(n)$ are the affine hyperplanes $H$ separating the alcoves $\tilde{w} A_{\circ}$ and $A_{\circ}$. These biject under the map $\tilde{w}^{-1}$ to the hyperplanes $\tilde{w}^{-1} H$ separating the alcoves $A_{\circ}$ and $\tilde{w}^{-1} A_{\circ}$. If $\tilde{w}=t_{r} w$, note that

$$
\tilde{w} H_{\alpha, k}=H_{w(\alpha), k+(r, \alpha)}
$$

This implies that the separating hyperplanes of $\tilde{w}$ orthogonal to the root $\alpha$ biject to the inversions of $\tilde{w}^{-1}$ orthogonal to the root $w^{-1}(\alpha)$. Then, for any alcove $A$ and positive root $\alpha \in \Phi^{+}$note that the number of Shi hyperplanes orthogonal to $\alpha$ that separate $A$ from $A_{\circ}$ is equal to 0 or 1 . Call this number $\chi_{\alpha}(A) \in\{0,1\}$. Finally, since the finite permutation $w^{-1}$ is a bijection on the roots $\Phi=\Phi^{+} \cup-\Phi^{+}$, this induces a bijection between the multisets $\left\{\chi_{\alpha}\left(\tilde{w} A_{\circ}\right): \alpha \in \Phi^{+}\right\}$and $\left\{\chi_{\alpha}\left(\tilde{w}^{-1} A_{\circ}\right): \alpha \in \Phi^{+}\right\}$. Hence these multisets have the same sum; i.e. $\operatorname{shi}(\tilde{w})=\operatorname{shi}^{-1}(\tilde{w})$.

For example, the affine permutation $\tilde{w}=[-2,2,6]$ has inversions $((2,4))$, $((3,4)),((3,5))$, and $((3,7))$, of which only $((3,7))$ does not come from a Shi hyperplane. The inverse $\tilde{w}^{-1}=[4,2,0]$ has inversions $((1,2)),((1,3))$, $((1,6))$, and $((2,3))$, where $((1,6))$ does not come from a Shi hyperplane. Hence $\operatorname{shi}(\tilde{w})=\operatorname{shi}^{-1}(\tilde{w})=3$.

\subsection{The inverse ish statistic}

Next we will compute a formula for the ish $^{-1}$ statistic. We will find that ish $^{-1}$ is a function on the root lattice $Q$.

To do this we need a lemma about the original ish statistic, which follows directly from Björner and Brenti [5, Lemma 4.2]. The proof is instructive, so we reproduce it here.

\footnotetext{
${ }^{2}$ This first sentence holds only for type $A$, but the rest of the proof holds for all crystallographic root systems.
} 
Lemma 4.4. Given an affine permutation $\tilde{w} \in \tilde{\mathfrak{S}}(n)$, choose $i \in\{1, \ldots, n\}$ such that $\tilde{w}(i)$ is maximum. Then

$$
\operatorname{ish}(\tilde{w})=\tilde{w}(i)-n .
$$

Proof. By definition, ish $(\tilde{w})$ is the number of affine tranpositions $((n, j))$ such that $n<j$ and $\tilde{w}^{I}(n)>\tilde{w}^{I}(j)$, where $\tilde{w}^{I}$ is the affine permutation defined by taking $\left[\tilde{w}^{I}(1), \ldots, \tilde{w}^{I}(n)\right]$ to be the increasing rearrangement of $[\tilde{w}(1), \ldots, \tilde{w}(n)]$. Setting $\tilde{u}=\tilde{w}^{I}$, we have $\operatorname{ish}(\tilde{w})=\operatorname{ish}(\tilde{u})$. We wish to show that $\operatorname{ish}(\tilde{u})=\tilde{u}(n)-n$.

So let $\tilde{u}=u+n r$, where $u \in \mathfrak{S}(n)$ is a finite permutation and $r \in Q$ is an element of the root lattice. Next fix an index $1 \leq i \leq n-1$ and consider the integer $\left\lfloor\frac{\tilde{u}(n)-\tilde{u}(i)}{n}\right\rfloor$. Since $\tilde{u}(1)<\cdots<\tilde{u}(n)$, this number is always nonnegative and it counts transpositions $((i, j))$ such that $n<j=i+k n$ (for some $k \in \mathbb{Z})$ and $\tilde{u}(n)>\tilde{u}(i+k n)$. Furthermore, since $-n \leq u(n)-u(i) \leq n$, note that

$$
\left\lfloor\frac{\tilde{u}(n)-\tilde{u}(i)}{n}\right\rfloor=\left\lfloor\frac{u(n)-u(i)}{n}+r(n)-r(i)\right\rfloor
$$

equals $r(n)-r(i)$ when $u(i)<u(n)$ and equals $r(n)-r(i)-1$ when $u(i)>$ $u(n)$. Finally, summing over $i$ gives

$$
\begin{aligned}
\operatorname{ish}(\tilde{u}) & =\sum_{i=1}^{n-1}\left\lfloor\frac{\tilde{u}(n)-\tilde{u}(i)}{n}\right\rfloor \\
& =\left(\sum_{i=1}^{n-1} r(n)-r(i)\right)-\#\{1 \leq i \leq n-1: u(i)>u(n)\} \\
& =(n-1) r(n)-\left(\sum_{i=1}^{n-1} r(i)\right)-(n-u(n)) \\
& =(n-1) r(n)-(0-r(n))-(n-u(n)) \\
& =(u(n)+n r(n))-n \\
& =\tilde{u}(n)-n .
\end{aligned}
$$

Happily, everything balances to create a simple formula. From this formula we get an expression for ish ${ }^{-1}$.

Theorem 4.5. Given an affine permutation $\tilde{w}=w+n r$, where $w \in \mathfrak{S}(n)$ is a finite permutation and $r \in Q$ is an element of the root lattice, choose the largest index $j \in\{1, \ldots, n\}$ such that the value of $r(j)$ is a minimum. Then

$$
\operatorname{ish}^{-1}(\tilde{w})=j+n(-r(j)-1) .
$$



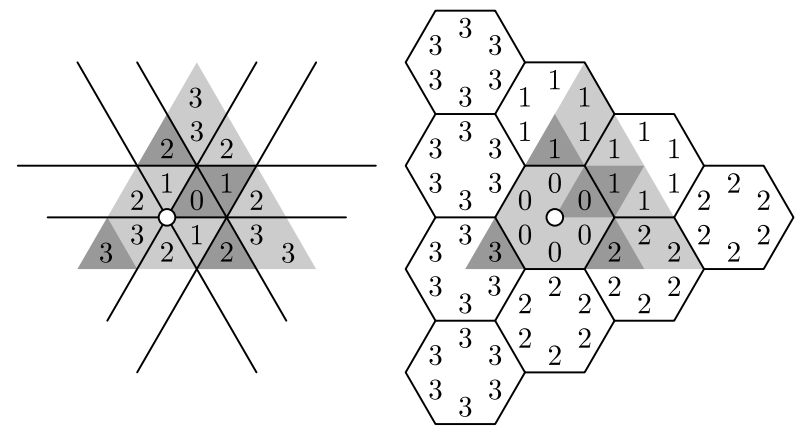

Figure 4.2: The shi and ish ${ }^{-1}$ statistics on $D^{4}(3)$.

Proof. For $i \in\{1, \ldots, n\}$ recall that $\tilde{w}^{-1}(i)=w^{-1}(i)-n r\left(w^{-1}(i)\right)$. Thus the largest value of $\tilde{w}^{-1}(i)$ over $i \in\{1, \ldots, n\}$ equals the largest value of $j-n r(j)$ over $j \in\{1, \ldots, n\}$. This value is achieved by the maximum $j$ such that $r(j)$ is a minimum. Lemma 4.4 then tells us that

$$
\operatorname{ish}^{-1}(\tilde{w})=\operatorname{ish}\left(\tilde{w}^{-1}\right)=(j-n r(j))-n .
$$

For example, Figure 2.2 displays the shi and ish $^{-1}$ statistics on the simplex $D^{4}(3)$. (The darker shaded alcoves have positive inverses.) This is the inverse of Figure 4.2.

Finally, we wish to emphasize the following. The value of ish $^{-1}(\tilde{w}=$ $\left.t_{r} w\right)$ depends only on the element $r \in Q$ of the root lattice. (This is the analogue of the fact that ish $\left(\tilde{w}=w_{I} \tilde{w}^{I}\right)$ depends only on the minimal coset representative $\tilde{w}^{I}$.) Combining this observation with the Main Theorem 3.6, we conclude that Haglund's bounce statistic is really a statistic on the root lattice of type $A$.

\section{Powers of nabla}

In this final section we will describe several ideas for future research, roughly in order of increasing generality. Most of this depends on the nabla operator $\nabla$ of F. Bergeron and Garsia [4], which we define first.

\subsection{The nabla operator}

We call a formal power series in $\mathbb{Q}\left[\left[x_{1}, x_{2}, \ldots,\right]\right]$ a symmetric function if it is invariant under permuting variables. Let $\Lambda=\oplus_{n \geq 0} \Lambda^{n}$ denote the ring 
of symmetric functions, graded by degree. Then $\Lambda^{n}$ is isomorphic to the vector space of (virtual) representations of the symmetric group $\mathfrak{S}(n)$ over $\mathbb{Q}$. Under this isomorphism, the role of the irreducible representations is played by the basis of Schur functions $s_{\lambda} \in \Lambda^{n}$, one for each partition $\lambda=$ $\left(\lambda_{1} \geq \lambda_{2} \geq \cdots\right)$ of the integer $n=\sum_{i} \lambda_{i}$.

If we extend the field of coefficients from $\mathbb{Q}$ to $\mathbb{Q}(q, t)$, another remarkable basis of $\Lambda^{n}$ is the set of modified Macdonald polynomials $\tilde{H}_{\mu}$, where again $\mu=\left(\mu_{1} \geq \mu_{2} \geq \cdots\right)$ is an integer partition of $n$. Let $\nu(\mu):=\sum_{i \geq 1}(i-1) \mu_{i},{ }^{3}$ and let $\mu^{\prime}$ be the conjugate partition defined by $\mu_{i}^{\prime}=\#\left\{j \geq 1: \mu_{j} \geq i\right\}$. Then the Bergeron-Garsia nabla operator is the unique $\mathbb{Q}(q, t)$-linear map on $\Lambda^{n}$ defined by

$$
\nabla\left(\tilde{H}_{\mu}\right)=q^{\nu\left(\mu^{\prime}\right)} t^{\nu(\mu)} \tilde{H}_{\mu} .
$$

That is, the modified Macdonald polynomials are a basis of eigenfunctions for $\nabla$. It turns out that many results on diagonal harmonics can be expressed elegantly in terms of $\nabla$. In particular, if $e_{n}=\sum_{i_{1}<\cdots<i_{n}} x_{i_{1}} \cdots x_{i_{n}}$ is the elementary symmetric function, then $\nabla\left(e_{n}\right)$ is the Frobenius character of the diagonal harmonics. That is, if we replace each Schur function in $\nabla\left(e_{n}\right)$ by its degree as a representation of $\mathfrak{S}(n)$, we obtain $\mathcal{D H}(n ; q, t)$ (in the notation of Section 1.1). For details, see Haglund [15].

Now we suggest some ways to generalize our earlier results, which amount to new conjectural interpretations of the $\nabla$ operator.

\subsection{Extended Shi arrangements}

Recall that the set of reflections in the affine Weyl group $\tilde{\mathfrak{S}}(n)$ is

$$
\tilde{T}=\{((i, j)): 1 \leq i \leq n, i<j\} .
$$

The affine transposition $((i, j))$ corresponds to the hyperplane $H_{\alpha, a}$ where $\alpha=e_{i^{\prime}}-e_{j^{\prime}}\left(\right.$ with $i^{\prime}=i-n(\lceil i / n\rceil-1), j^{\prime}=j-n(\lceil j / n\rceil-1)$, and $a=$ $\left.\left\lceil\frac{i}{n}\right\rceil-\left\lceil\frac{j}{n}\right\rceil\right)$. We will call $\left\lfloor\frac{j-i}{n}\right\rfloor=k$ the height of the hyperplane; this is some measure of how far the hyperplane is from the fundamental alcove. Recall that the Shi arrangement consists of the hyperplanes of height 0 . We may now define the $m$-extended Shi arrangement.

Definition 5.1. Let Shi ${ }^{m}(n)$ denote the set of affine transpositions $((i, j))$ with height in the set $\{0, \ldots, m-1\}$. Equivalently,

$$
\operatorname{Shi}^{m}(n):=\left\{e_{i}-e_{j}=a: 1 \leq i<j \leq n, a \in\{-m+1, \ldots, m\}\right\} .
$$

\footnotetext{
${ }^{3}$ The notation $n(\mu)$ is also used.
} 
Athanasiadis proved [3, Proposition 3.5] that every chamber of $\mathrm{Shi}^{m}(n)$ contains a unique alcove of minimum length. Fishel and Vazirani [7, Theorem 7.1] proved that the inverses of these representing alcoves are precisely the alcoves contained in the simplex $D^{m n+1}(n) \subseteq \mathbb{R}_{0}^{n}$ bounded by the hyperplanes

$$
\left\{e_{i}-e_{i+1}=-m-1: 1 \leq i \leq n-1\right\} \cup\left\{e_{1}-e_{n}=m+1\right\}
$$

Sommers showed in [27, proof of Theorem 5.7] that the simplex $D^{m n+1}(n)$ is congruent to the dilation $(m n+1) A_{\circ}$ of the fundamental alcove $A_{\circ}$. Since this occurs in the $(n-1)$-dimensional space $\mathbb{R}_{0}^{n}$, the simplex $D^{m n+1}(n)$ consists of $(m n+1)^{n-1}$ alcoves. We would like to extend the statistics shi and ish ${ }^{-1}$ to these alcoves.

This turns out to be very easy to do. The shi statistic generalizes naturally, and the ish statistic needs no generalization at all.

Definition 5.2. Given an affine permutation $\tilde{w} \in \tilde{\mathfrak{S}}(n)$, let $\operatorname{shi}^{m}(\tilde{w})$ denote the number of hyperplanes of $\operatorname{Shi}^{m}(n)$ separating $\tilde{w} A_{\circ}$ from the fundamental alcove $A_{\circ}$.

The proof of Theorem 4.3 generalizes to show that $\operatorname{shi}^{m}(\tilde{w})=\operatorname{shi}^{m}\left(\tilde{w}^{-1}\right)$ for all $\tilde{w} \in \tilde{\mathfrak{S}}(n)$ (we simply let $\chi_{\alpha}(A)$ take values beyond just 0 and 1 ). Thus we want to study the joint distribution of $\mathrm{shi}^{m}$ and ish ${ }^{-1}$ on the alcoves of the simplex $D^{m n+1}(n)$.

Conjecture 5.3. Consider the following generating function for $\mathrm{shi}^{m}$ and ish $^{-1}$ over alcoves in the dilated simplex $D^{m n+1}(n)$,

$$
\mathrm{Shi}^{m}(n ; q, t):=\sum_{A \subseteq D^{m n+1}(n)} q^{\mathrm{ish}^{-1}(A)} t^{m\left(\begin{array}{c}
n \\
2
\end{array}\right)-\operatorname{shi}^{m}(A)},
$$

and let $\mathrm{Shi}_{+}^{m}(n ; q, t)$ denote the same sum over alcoves whose inverses are in the dominant cone $C_{\circ}$. We conjecture the following:

(1) $\mathrm{Shi}^{m}(n ; q, t)$ is the Hilbert series of $\nabla^{m}\left(e_{n}\right)$.

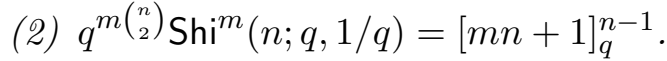

(3) $\mathrm{Shi}_{+}^{m}(n ; q, t)$ is the Hilbert series for the sign-isotypic component of $\nabla^{m}\left(e_{n}\right)$.

(4) $q^{m\left(\begin{array}{c}n \\ 2\end{array}\right)} \operatorname{Shi}_{+}^{m}(n ; q, 1 / q)=\frac{1}{[n]_{q}}\left[\begin{array}{c}(m+1) n \\ n-1\end{array}\right]_{q}$, the $q$-Fuss-Catalan number.

For example, let $m=2$ and $n=3$. Figure 5.1 displays the statistics shi ${ }^{2}$ and ish $^{-1}$ on the alcoves of $D^{7}(3)$, and Table 1 displays the corresponding 

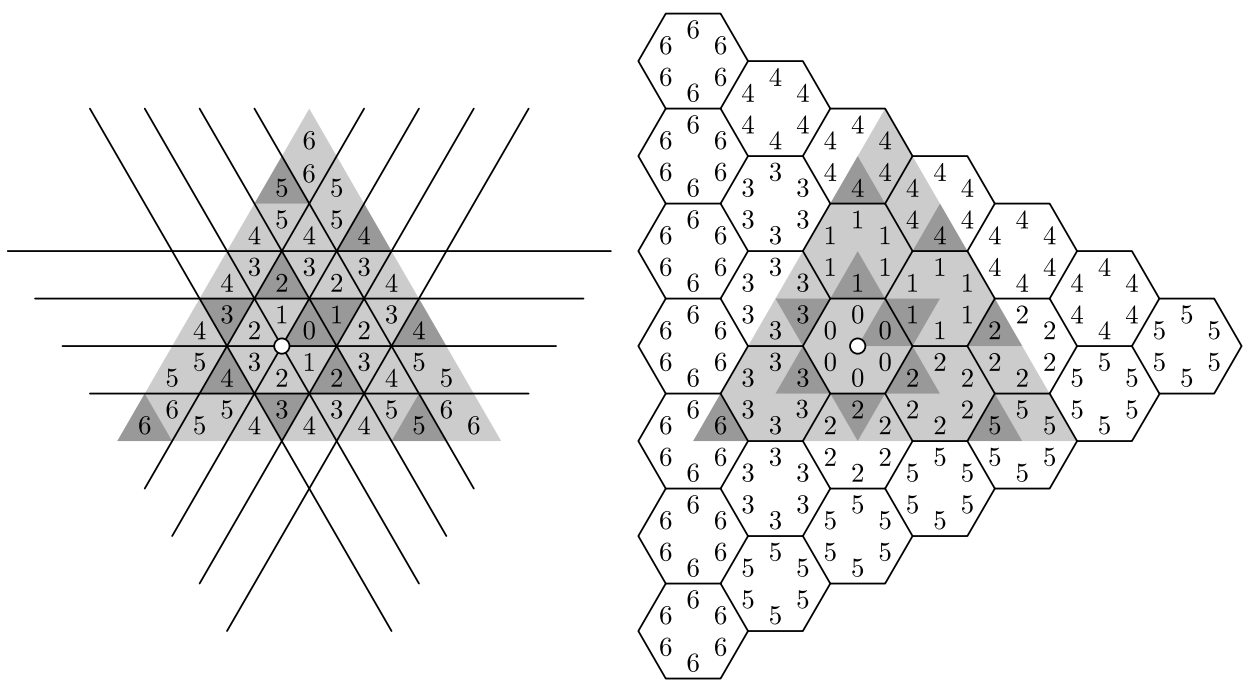

Figure 5.1: The $\mathrm{shi}^{2}$ and ish ${ }^{-1}$ statistics on $D^{7}(3)$.

Table 1: The generating functions $\operatorname{Shi}^{2}(3: q, t)$ and $\operatorname{Shi}_{+}^{2}(3 ; q, t)$

\begin{tabular}{cc|ccccccc} 
& \multicolumn{8}{|c}{ ish $^{-1}$} \\
& & 0 & 1 & 2 & 3 & 4 & 5 & 6 \\
\hline & 0 & 1 & & & & & & \\
& 1 & 2 & 1 & & & & & \\
shi $^{2}$ & 2 & 2 & 3 & 1 & & & & \\
& 3 & 1 & 4 & 3 & 1 & & & \\
& 4 & & 3 & 5 & 3 & 1 & & \\
& 5 & & 1 & 3 & 4 & 3 & 1 & \\
& 6 & & & & 1 & 2 & 2 & 1
\end{tabular}

\begin{tabular}{cc|ccccccc} 
& & \multicolumn{1}{c}{ ish $^{-1}$} & & & \\
& & 0 & 1 & 2 & 3 & 4 & 5 & 6 \\
\hline & 0 & 1 & & & & & & \\
& 1 & & 1 & & & & & \\
$\operatorname{shi}^{2}$ & 2 & & 1 & 1 & & & & \\
& 3 & & & 1 & 1 & & & \\
& 4 & & & 1 & 1 & 1 & & \\
& 5 & & & & & 1 & 1 & \\
& 6 & & & & & & & 1
\end{tabular}

generating functions. One may observe that all four assertions hold in this case.

Positive powers of $\nabla$ have been well-studied. We believe that a suitable extension of our Main Theorem 3.6 is possible, which would make our conjectures equivalent to earlier conjectures of Haiman, Loehr and Remmel (see [19]), which are based on lattice paths from $(0,0)$ to $(m n, n)$ that stay weakly above the diagonal $y=x / m$.

\subsection{Bounded chambers}

While positive powers of $\nabla$ have been investigated by several authors, to our knowledge there has been no combinatorial conjecture for negative powers of $\nabla$. In this section we will provide one. 
It was shown by Edelman and Reiner [6, Section 3] and by Postnikov and Stanley [22, Proposition 9.8] that the characteristic polynomial of the $m$-extended Shi arrangement is

$$
\chi\left(\operatorname{Shi}^{m}(n), x\right)=(x-m n)^{n-1} .
$$

Hence, by Zaslavsky's Theorem, Shi ${ }^{m}(n)$ has $(m n+1)^{n-1}$ chambers (as we noted above), and it has $(m n-1)^{n-1}$ bounded chambers. Athanasiadis showed that this is also an example of Ehrhart reciprocity [2].

As mentioned earlier, Athanasiadis showed that each chamber of $\mathrm{Shi}^{m}(n)$ contains a unique alcove of minimum length. In the case $m=1$, Sommers showed [27, Lemmas 5.1 and 5.2] that, moreover, every bounded chamber of $\mathrm{Shi}^{m}(n)$ contains a unique alcove of maximum length. Fishel and Vazirani [8, Theorem 6.1] showed that this is true for general $m \geq 1$, and furthermore that the inverses of these alcoves are precisely the alcoves contained in the simplex $D^{m n-1}(n) \subseteq \mathbb{R}_{0}^{n}$ bounded by the hyperplanes

$$
\left\{e_{i}-e_{i+1}=m: 1 \leq i \leq n-1\right\} \cup\left\{e_{1}-e_{n}=-m+1\right\} \text {. }
$$

As with $D^{m n+1}(n)$ above, Sommers has shown that $D^{m n-1}(n)$ is congruent to the dilation $(m n-1) A_{\circ}$ of the fundamental alcove, which implies that $D^{m n-1}(n)$ contains $(m n-1)^{n-1}$ alcoves. We wish to study the statistics shi ${ }^{m}$ and ish $^{-1}$ on these alcoves.

Conjecture 5.4. Consider the following generating function for shi ${ }^{m}$ and ish $^{-1}$ over alcoves in the dilated simplex $D^{m n-1}(n)$,

$$
\operatorname{Shi}^{-m}(n ; q, t):=\sum_{A \subseteq D^{m n-1}(n)} q^{\mathrm{ish}^{-1}(A)} t^{(m n-2)(n-1) / 2-\operatorname{shi}^{m}(A)},
$$

and let $\mathrm{Shi}_{+}^{-m}(n ; q, t)$ denote the same sum over alcoves whose inverses are in the dominant cone $C_{\circ}$. We conjecture the following.

(1) $(-1)^{n-1} \mathrm{Shi}^{-m}(n ; 1 / q, 1 / t) / q^{n-1} t^{n-1}$ is the Hilbert series of $\nabla^{-m}\left(e_{n}\right)$.

(2) $q^{(m n-2)(n-1) / 2} \mathrm{Shi}^{-m}(n ; q, 1 / q)=[m n-1]_{q}^{n-1}$.

(3) $(-1)^{n-1} \mathrm{Shi}_{+}^{-m}(n ; 1 / q, 1 / t) / q^{n-1} t^{n-1}$ is the Hilbert series for the signisotypic component of $\nabla^{-m}\left(e_{n}\right)$.

(4) $q^{(m n-2)(n-1) / 2} \mathrm{Shi}_{+}^{-m}(n ; q, 1 / q)=\frac{1}{[n]_{q}}\left[\begin{array}{c}(m+1) n-2 \\ n-1\end{array}\right]_{q}$.

For example, Figure 5.2 displays the statistics $\operatorname{shi}^{2}$ and ish $^{-1}$ on the simplex $D^{5}(3)$, and Table 2 displays the corresponding generating functions. One may observe that all four assertions hold for this data. 

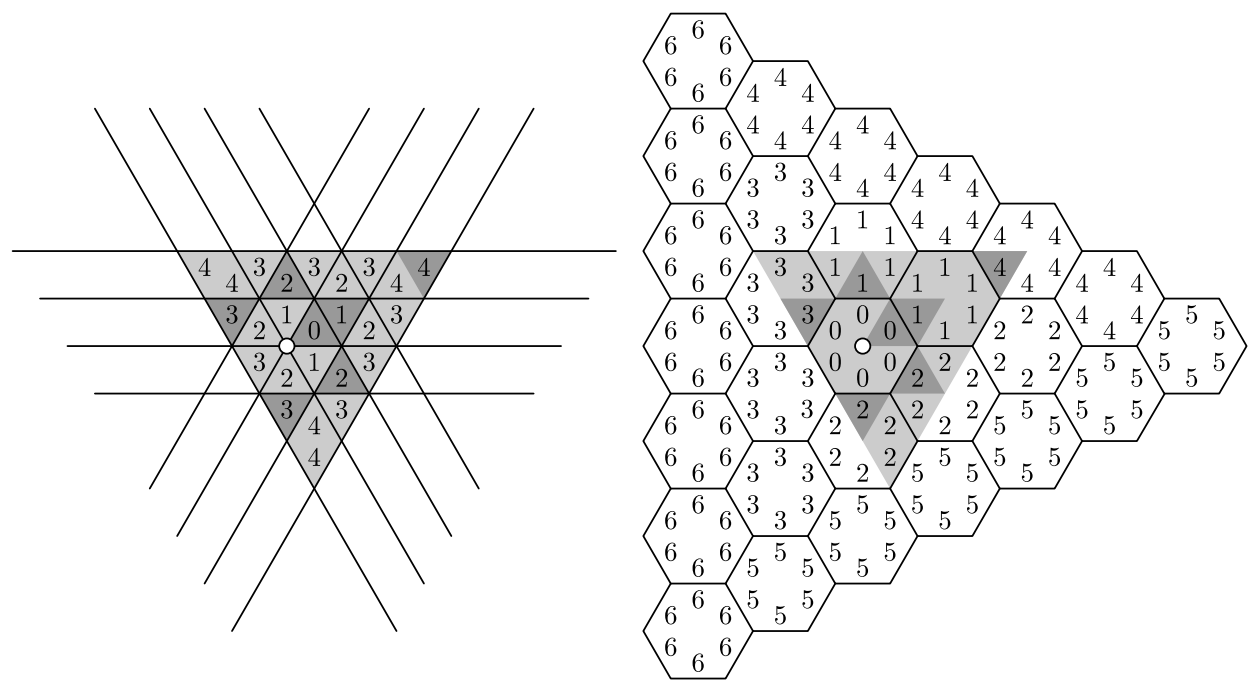

Figure 5.2: The $\operatorname{shi}^{2}$ and ish $^{-1}$ statistics on $D^{5}(3)$.

Table 2: The generating functions $\operatorname{Shi}^{-2}(3: q, t)$ and $\mathrm{Shi}_{+}^{-2}(3 ; q, t)$

\begin{tabular}{cc|ccccc}
\multicolumn{1}{c}{} & \multicolumn{7}{|c}{ ish $^{-1}$} \\
& & 0 & 1 & 2 & 3 & 4 \\
\hline & 0 & 1 & & & & \\
& 1 & 2 & 1 & & & \\
shi $^{2}$ & 2 & 2 & 3 & 1 & & \\
& 3 & 1 & 4 & 3 & 1 & \\
& 4 & & 1 & 2 & 2 & 1
\end{tabular}

\begin{tabular}{cc|ccccc} 
& & \multicolumn{6}{|c}{ ish $^{-1}$} \\
& & 0 & 1 & 2 & 3 & 4 \\
\hline & 0 & 1 & & & & \\
& 1 & & 1 & & & \\
shi $^{2}$ & 2 & & 1 & 1 & & \\
& 3 & & & 1 & 1 & \\
& 4 & & & & & 1
\end{tabular}

Combining Conjectures 5.3 and 5.4, we obtain a conjectural combinatorial interpretation for all integral powers of the nabla operator acting on $e_{n}$. We wonder whether Athanasiadis' result [2] on Ehrhart reciprocity for Shi arrangements may reflect some sort of reciprocity theorem for the nabla operator.

\subsection{Interpolation}

Since the forms of Conjectures 5.3 and 5.4 are so similar, one may ask for a formula encompassing them both. In this case we do not have a concrete conjecture, but we we will suggest some ideas.

The simplices $D^{m n+1}(n)$ and $D^{m n-1}(n)$ are both special cases of the following construction of Sommers: 
Recall that the root system of type $A_{n-1}$ is defined by $\Phi=\left\{e_{i}-e_{j}: 1 \leq\right.$ $i, j \leq n\}$, and the basis of simple roots is $\Delta=\left\{e_{i}-e_{i+1}: 1 \leq i \leq n-1\right\}$. Given a root $\alpha \in \Phi$, let $b$ denote the sum of its coefficients in the simple root basis; we say that $b$ is the height of the root $\alpha$. Let $\Phi_{b} \subseteq \Phi$ denote the set of roots of height $b$. Finally, let $p=a n+b$ be any integer coprime to $n$, with $1 \leq b \leq n-1$, and let $D^{p}(n)$ be the region containing the origin and bounded by the hyperplanes

$$
\left\{H_{\alpha, a}: \alpha \in \Phi_{-b}\right\} \cup\left\{H_{\alpha, a+1}: \alpha \in \Phi_{n-b}\right\}
$$

As with $D^{m n+1}(n)$ and $D^{m n-1}(n)$, Sommers showed for any $p$ coprime to $n$ that $D^{p}(n)$

- is congruent to a dilation $p A_{\circ}$ of the fundamental alcove;

- contains $p^{n-1}$ alcoves; and

- contains $\frac{1}{p+n}\left(\begin{array}{c}p+n \\ n\end{array}\right)$ alcoves whose inverses are in the dominant cone.

We suggest the following:

Open Problem. Define a statistic stat on the alcoves of $D^{p}(n)$. Consider the generating function

$$
F(p, n ; q, t):=\sum_{A \subseteq D^{p}(n)} q^{\operatorname{ish}^{-1}(A)} t^{(p-1)(n-1) / 2-\operatorname{stat}(A)},
$$

and let $F_{+}(p, n ; q, t)$ denote the same sum over alcoves whose inverses lie in the dominant cone $C_{\circ}$. These generating functions should satisfy

(1) $F(p, n ; q, t)=F(p, n ; t, q)$.

(2) $q^{(p-1)(n-1) / 2} F(p, n ; q, 1 / q)=[p]_{q}^{n-1}$.

(3) $q^{(p-1)(n-1) / 2} F(p, n ; q, 1 / q)=\frac{1}{[p+n]_{q}}\left[\begin{array}{c}p+n \\ n\end{array}\right]_{q}$.

Note that ish ${ }^{-1}$ does not need to be modified. It is the shi statistic that is difficult to define in general. We note that the smallest mystery case is $p=2$ and $n=5$, which corresponds to the 4-dimensional simplex $D^{2}(5)$ in $\mathbb{R}_{0}^{5}$ bounded by the hyperplanes

$$
e_{1}-e_{3}=e_{2}-e_{4}=e_{3}-e_{5}=0 \quad \text { and } \quad e_{1}-e_{4}=e_{2}-e_{5}=1
$$

This simplex contains $2^{4}=16$ alcoves, corresponding to the affine permutations 


$$
\begin{array}{cccc}
{[-1,2,5,3,6],} & {[0,3,2,4,6],} & {[1,2,4,3,5],} & {[2,1,3,4,5],} \\
{[0,2,3,4,6],} & {[2,0,3,6,4],} & {[1,3,2,4,5],} & {[2,1,3,5,4],} \\
{[0,2,4,3,6],} & {[1,2,3,4,5],} & {[1,3,2,5,4],} & {[2,1,4,3,5],} \\
{[0,3,1,4,6],} & {[1,2,3,5,4],} & {[1,4,2,5,3],} & {[3,1,4,2,5] .}
\end{array}
$$

Of these, only $\frac{1}{2+5}\left(\begin{array}{c}2+5 \\ 5\end{array}\right)=3$ have inverses in the dominant cone - namely, $[1,2,3,4,5],[0,2,3,4,6]$ and $[2,0,3,6,4]$. The distribution of ish $^{-1}$ over the former 16 is $\sum q^{\text {ish }}{ }^{-1}(A)=10+5 q+q^{2}$ and the distribution of ish ${ }^{-1}$ over the latter 3 is $\sum q^{\text {ish }^{-1}(A)}=1+q+q^{2}$. We do not know what the analogue of shi is in this case.

\subsection{Other types}

In this paper we have focused on the affine Weyl group of type $\tilde{A}_{n}$, which is the group $\tilde{\mathfrak{S}}(n)$ of affine permutations. However, we have tried to use language throughout that is general to all affine Weyl groups. Certainly, the combinatorics of Shi arrangements is completely general. Also, Sommers' simplex $D^{p}(h)$ is defined in general for any integer $p$ coprime to the Coxeter number $h$.

Haiman observed that the most obvious generalization of the ring of harmonic polynomials to other types is "too large" (see [13, Section 7]), and he conjectured that some suitable quotient should be considered instead. Using rational Cherednik algebras, Gordon [11] was able to construct such a quotient. Gordon and Griffeth [12] have now observed that this module does have a suitable bigrading and it satisfies many of the desired combinatorial properties. Gordon-Griffeth [12] and Stump [30] have both defined $q, t$-Catalan numbers in general type (even in complex types), however their numbers disagree in the non-well-generated complex types. This is an active area, and we wish to emphasize: as of this writing, there is no known combinatorial interpretation for these objects beyond type $A$.

We suggest that the shi statistic on the simplex $D^{h+1}(h)$ is a good place to start. The next step is to define an analogue of the ish ${ }^{-1}$ statistic. Unfortunately, we have checked that in type $B_{2}$ it cannot simply be a statistic on the root lattice.

\section{Acknowledgements}

The author thanks Christos Athanasiadis, Steve Griffeth, Brant Jones, Nick Loehr, Eric Sommers, and Greg Warrington for helpful discussions. Extra thanks are due to Eric Sommers for suggesting the proof of Theorem 4.3 
and to Jim Haglund (via Greg Warrington) for providing Maple code for working with $\nabla$. The original idea for this work was inspired by discussions with Steve Griffeth and by the paper [7] of Fishel and Vazirani.

In January 2010 we learned that Mark Haiman had a result in 2007 (unpublished) equivalent to our Main Theorem 3.6. Hopefully this will appear in a future paper.

\section{References}

[1] D. Armstrong and B. Rhoades (2012). The Shi arrangement and the Ish arrangement. Trans. Amer. Math. Soc. 364(3) 1509-1528. MR2869184

[2] C. A. Athanasiadis (2010). A combinatorial reciprocity theorem for hyperplane arrangements. Canad. Math. Bull. 53 3-10. MR2583206

[3] C. A. Athanasiadis (2005). On a refinement of the generalized Catalan numbers for Weyl groups. Trans. Amer. Math. Soc. 357 179-196. MR2098091

[4] F. Bergeron and A. Garsia (1999). Science fiction and Macdonald polynomials. CRM Proceedings and Lecture Notes AMS VI 3 363-429.

[5] A. Björner and F. Brenti (1996). Affine permutations of type A. Electric Journal of Combinatorics 3(2) \# R18. MR1392503

[6] P. H. Edelman and V. Reiner (1996). Free arrangements and rhombic tilings. Discrete Comput. Geom. 15 307-340. MR1380397

[7] S. Fishel and M. Vazirani (2010). A bijection between dominant Shi regions and core partitions. European J. Combin. 31(8) 2087-2101. MR2718283

[8] S. Fishel and M. Vazirani. A bijection between (bounded) dominant Shi regions and core partitions. In: DMTCS Proceedings, 22nd International Conference on Formal Power Series and Algebraic Combinatorics (FPSAC 2010). MR2673843

[9] A. M. Garsia and J. Haglund (2001). A positivity result in the theory of Macdonald polynomials. Proc. Nat. Acad. Sci. U.S.A. 98 4313-4316. MR1819133

[10] A. M. Garsia and J. Haglund (2002). A proof of the $q$, t-Catalan positivity conjecture. Discrete Math. 256 677-717. MR1935784

[11] I. Gordon (2003). On the quotient ring by diagonal coinvariants, Invent. Math. 153 503-518. MR2000467 
[12] S. Griffeth and I. Gordon. Catalan numbers for complex reflection groups. arXiv:0912.1578.

[13] M. Haiman (1994). Conjectures on the quotient ring by diagonal invariants. J. Algebraic Combin. 3(1) 17-76. MR1256101

[14] J. Haglund (2003). Conjectured statistics for the $q, t$-Catalan numbers. Adv. Math. 175(2) 319-334. MR1972636

[15] J. Haglund (2008). The q,t-Catalan numbers and the space of diagonal harmonics, University Lecture Series, Vol. 41. American Mathematical Society, Providence, RI. MR2371044

[16] J. Haglund and N. Loehr (2005). A conjectured combinatorial formula for the Hilbert series for Diagonal Harmonics. Discrete Math. (Proc. of the FPSAC 2002 Conference held in Melbourne, Australia) 298189 204. MR2163448

[17] N. A. Loehr (2005). Combinatorics of $q, t$-parking functions. Adv. in Appl. Math. 34(2) 408-425. MR2110560

[18] N. A. Loehr (2007). The major index specialization of the $q, t$-Catalan. Ars. Combin. 83 145-160. MR2305754

[19] N. A. Loehr and J. Remmel (2004). Conjectured combinatorial models for the Hilbert series of generalized diagonal harmonics modules. Electron. J. Combin. 11(research paper R68) 64 pp. MR2097334

[20] N. A. Loehr and G. Warrington (2008). Nested quantum Dyck paths and $\nabla\left(s_{\lambda}\right)$. International Mathematics Research Notices 2008 Article ID rnm157. MR2418288

[21] G. Lusztig (1983). Some examples of square integrable representations of semisimple p-adic groups. Trans. Amer. Math. Soc. 277 623-653. MR0694380

[22] A. Postnikov and R. P. Stanley (2000). Deformations of Coxeter hyperplane arrangements. J. Combin. Theory Ser. A 91 544-597. MR1780038

[23] J.-Y. Shi (1986). The Kazhdan-Lusztig Cells in Certain Affine Weyl groups. Lecture Notes in Math., Vol. 1179, Springer-Verlag. MR0835214

[24] J.-Y. Shi (1987). Alcoves corresponding to an affine Weyl group. $J$. London Math. Soc. (2) 35(1) 42-55. MR0871764

[25] J.-Y. Shi (1987). Sign types corresponding to an affine Weyl group. J. London Math. Soc. (2) 35(1) 56-74. MR0871765 
[26] J.-Y. Shi (1997). The number of $\oplus$-sign types. Quarterly J. Math., Oxford 48 93-105. MR1439701

[27] E. Sommers (2005). B-stable ideals in the nilradical of a Borel subalgebra. Canad. Math. Bull. 48(3) 460-472. MR2154088

[28] R. P. Stanley (1996). Hyperplane arrangements, interval orders, and trees. Poc. Nat. Acad. Sci. 93 2620-2625. MR1379568

[29] R. P. Stanley (2007). An introduction to hyperplane arrangements. In: Geometric Combinatorics, IAS/Park City Math. Ser. 13, Amer. Math. Soc., Providence, RI. MR2383131

[30] C. Stump. q,t-Fuss-Catalan numbers for finite reflection groups, to appear in Journal of Algebraic Combinatorics, arXiv:0901.1574. MR2657714

[31] H. Weyl (1936). The Classical Groups: Their Invariants and Representations. Princeton University Press; 2nd ed., 1946. MR1488158

Drew Armstrong

Department of Mathematics

UNIVERSITY OF MIAMI

Coral Gables, FL 33146

USA

E-mail address: armstrong@math.miami.edu

ReCEIVED June 12, 2012 